\title{
Models of mesoscopic time-resolved current detection
}

\author{
Adam Bednorz ${ }^{1,2}$ and Wolfgang Belzig ${ }^{1}$ \\ ${ }^{1}$ Fachbereich Physik, Universität Konstanz, D-78457 Konstanz, Germany \\ ${ }^{2}$ University of Warsaw, Hoża 69, PL-00681 Warsaw, Poland
}

(Dated: November 7, 2018)

\begin{abstract}
Quantum transport in mesoscopic conductors is essentially governed by the laws of quantum mechanics. One of the major open questions of quantum mechanics is what happens if non-commuting observables are measured simultaneously. Since current operators at different times do not commute, the high-frequency correlation functions of the current are realization of this fundamental quantum question. We formulate this problem in the context of measurements of finite-frequency current cumulants in a general quantum point contact, which are the subject to ongoing experimental effort. To this end, we present two models of detectors that correspond to a weak time-resolved measurement of the electronic current in a mesoscopic junction. In both cases, the backaction of the detector leads to observable corrections to the current correlations functions involving the so-called noise susceptibilities. As a result, we propose a reinterpretation of environmental corrections to the finite-frequency cumulants as inevitable effect resulting from basic quantum mechanical principles. Finally we make concrete predictions for the temperature-, voltage-, and frequency-dependence of the third cumulant, which could be verified directly using current experimental techniques.
\end{abstract}

\section{INTRODUCTION}

Since the advent of mesoscopic physics, the quantum mechanical properties of charge transport in conductors are under intensive research. Most manifestations to date are concerned with properties following from the quantum mechanical wave nature $\frac{1}{}$ More recently the quantum mechanics of the electron spin has been investigated in electron transport. 2 On the other hand, the aspect of measurement has attracted less attention so far in quantum transport. This is surprising in view of it's eminent importance for the fundamental difference to classical physics. $\underline{3}$

The electronic transport through mesoscopic junctions is quite well described by independent electrons. ${ }^{\underline{4}}$ Due to the Landauer formula, the conductance can be expressed in terms of the single-particle scattering matrix and the level occupations of the leads. The same scattering matrix can describe low-frequency current noise. $\underline{\underline{4}}$ Experimentally, the scattering description has been confirmed in the case of low frequency noise $\mathrm{F}^{\frac{5}{4}}$ a while ago.

It is remarkable, that the same scattering matrix also describes higher order correlation functions of current leading to the so-called full counting statistics (FCS) $\underline{6}$ These results have been confirmed for the third cumulant of tunnel junctions only, so far $\stackrel{8,9}{ }$ However, a proper account of the first experiments showed that the measured third cumulant was governed by an environmental contribution. 10

In the last years it has also been realized, that the measurement of the quantum noise depends in an essential way on the detection scheme. ${ }^{11}$ To understand this, one considers different Fourier transform of the currentcurrent correlation function $C\left(t, t^{\prime}\right)=\left\langle\hat{I}(t) \hat{I}\left(t^{\prime}\right)\right\rangle$. It turns out that the unsymmetrized correlators $C(\omega)=$ $\int d \tau C(0, \tau) \exp ( \pm i \omega \tau)$ are related to absorption or emission of an energy quantum $\hbar \omega$, respectively, and can therefore be measured in a corresponding detector. On the other hand, a classical detector measures always the real combination $C(\omega)+C(-\omega)$, corresponding to the anti-commutator of the current operators. The high frequency noise has been also tested experimentally. $12-14$ The results are in full agreement with the scattering theory of non-interacting quasiparticles in mesoscopic conductors.

From the above it is clear that a time-resolved detection of the quantum mechanical current in a quantum transport process has to properly account for the quantum rules for measurements. This is not so much an experimental question - since experimentalists automatically obey the rules of quantum mechanics - rather, the proper treatment of a quantum measurement represents a challenge to theorists, who have to worry, which expectation values are actually measurable. Hence, the extension of full counting statistics to time-resolved (highfrequency) correlations requires first a proper definition in terms of projective measurement. $\underline{3}$ Qualitatively, one can distinguish two extremes. A continuous projective measurement would lead up in suppression of the dynamics of the system - a phenomenon known as quantum Zeno effect. 15 To avoid the Zeno effect it is necessary to include the detector's degrees of freedom into the complete evolution. Some effects of the detector backaction have been addressed already in the literature. ${ }^{16-19}$ However, instead of including a specific detector's dynamics one can restrict the treatment to the system only by replacing the projection by so-called Kraus operators 20 These define a positive operator-valued measure $(\mathrm{POVM})^{21}$ and by virtue of Naimark's theorem the two methods are equivalent ${ }^{22}$ as long as initially the detector and the system are uncorrelated..$^{23}$

Once we have established the detection model we can again express current correlations of arbitrary order in terms of the single-particle scattering matrix. However, it turns out that a simple evaluation of these averages quickly leads to very complicated and cumbersome ex- 
pressions. Nevertheless, the demanding task necessary to analyze the generating functional leads to interesting analytic results for the frequency-dependent cumulants and the full counting statistics $\stackrel{24}{\underline{26}}$ Below, we will provide a simplified framework, which allows to evaluate correlators of arbitrary order in an efficient way.

An important recent development is that the third cumulant has been measured at high frequency. ${ }^{27}$ These quite remarkable results show interesting quantum features at $e V=\hbar \omega$. However, most of this feature can be explained by environmental effects due to a series impedance. These result in a mixing of the thirdorder correlator with the noise susceptibilities due to the frequency-dependent backaction of an environmental impedance. After fitting the spurious contribution, the resulting third cumulant of the considered tunnel junction shows no frequency dispersion at all. This is in perfect accordance with the theory, which predicts in fact a dispersionless third cumulant in the case of a tunnel junction ${ }^{24}$ However, it is most likely just a question of time until the third cumulant for a mesoscopic conductor with non-opaque channel is measured. Hence, for these experiments it is vital to distinguish the environmental contribution from the quantum mechanical backaction of the detector.

Our findings can be summarized in two main achievements. On one hand, most of the article is devoted to the development of concrete models of mesoscopic detectors of high-frequency current-detection. The interplay between the quantum mechanical projection and backaction plays here a major role and we clarify below, how this can or cannot be distinguished from the environmental effect. On the other hand, we present an efficient method to calculate higher order correlation functions of the current in mesoscopic junctions. This allows us to present several results for the third cumulant and the noise susceptibility in a unified and transparent manner. The method can be the basis for an efficient evaluation of fourth and higher order correlators, which is important for example in photon counting statistics, ${ }^{28,29}$

In the main body of the article we are going to construct a model of a projective time-resolved measurement of the current in a mesoscopic conductor. We present two possibilities. One is to define a POVM, which corresponds to a weak measurement of the current in a quantum point contact. This phenomenological and very simple approach has the appeal that the competition between projection and weak measurement is qualitatively reflected. However, a concrete physical realization is difficult to find. Another possibility is based on a physical model of a detector, which we call quantum tape. Our detector will be a massless bosonic field described by charge density and current operators, parametrized by a one-dimensional variable. It is equivalent to the noninteracting Schwinger-Tomonaga-Luttinger model of a one-dimensional fermionic field $\stackrel{30}{0}$ Equivalently the detector can be thought of as a transmission line, which is described by the same Hamiltonian. This detector model is actually a massless version of a Josephson transmission line detector $\stackrel{18}{\underline{10}}$ The field can be decomposed into left and right going components with a constant velocity. In this way, the spatial coordinate of the detector, which is coupled to the system only at one point in space, corresponds effectively to a time-coordinate. The information registered by the detector is moved away from the system due to the internal dynamics of the detector. Finally a spatially resolved strong projection is applied to the detector at one instant of time, and the result can be translated into the temporal current profile. The projection can be made on one or both ends of the tape due to its bidirectional information transfer. The results always depend on the detection scheme. In the limit of weak coupling the detector's contribution is only a large Gaussian offset noise, independent of the measured system. ${ }^{31}$ Accordingly, the influence of the backaction of the detector onto the system is also weak but always present.

The secondary goal of the present article is to develop a compact method to calculate arbitrary cumulants of current operators in a junction with energy-independent transmission. To this end, we set up a current algebra, which is especially suited for non-equilibrium scattering problems. This becomes necessary, when considering different orderings of higher-order correlators at finite frequency. A straightforward evaluation using the scattering states is possible, but becomes in general very cumbersome. Introducing an algebra for certain current operators of a quantum point contact greatly simplifies this calculation and will therefore be useful also in other context, hopefully. In the respective limits, our results are, of course, in agreement with existing results.

The paper is organized as follows. We begin with the description of the mesoscopic junction in Sec. II, together with an overview of necessary mathematical tools. In Sec. III we present the POVM detection model and extend it to many detectors. In this model, we are able to derive also some higher cumulants of current in the limit of weak coupling between the detector and the system. In certain limits (weak transmission or reflection) one can find the complete FCS generating functional. Finally in Sec. IV, we present a strictly projective measurement model - the quantum tape, which gives results similar to the POVM. Many useful but lengthy mathematical details are moved to appendices.

\section{MESOSCOPIC JUNCTION}

The junction is defined as constriction in a two dimensional electron gas, which is narrow in the $y$-direction and relatively long in the $x$-direction. We assume no electron-electron interactions and no magnetic field. The 
Hamiltonian of the system reads

$$
\begin{aligned}
& \hat{H}=\sum_{\sigma} \int d x d y\left[-\hat{\psi}_{\sigma}^{\dagger}(x, y) \hbar^{2} \Delta \hat{\psi}_{\sigma}(x, y) / 2 m\right. \\
& \left.+e V_{e}(x, y, t) \hat{\psi}_{\sigma}^{\dagger}(x, y) \hat{\psi}_{\sigma}(x, y)\right]
\end{aligned}
$$

The fermionic operators satisfy standard anticommutation relations

$$
\begin{aligned}
& \left\{\hat{\psi}_{\sigma}(x, y), \hat{\psi}_{\sigma^{\prime}}\left(x^{\prime}, y^{\prime}\right)\right\}=0 \\
& \left\{\hat{\psi}_{\sigma}(x, y), \hat{\psi}_{\sigma^{\prime}}^{\dagger}\left(x^{\prime}, y^{\prime}\right)\right\}=\delta_{\sigma \sigma^{\prime}} \delta\left(x-x^{\prime}\right) \delta\left(y-y^{\prime}\right) .
\end{aligned}
$$

The external potential splits into three parts

$$
V_{e}(x, y, t)=V_{x}(x)+V_{y}(y)+V(x, t),
$$

where $V_{x}$ is the scattering potential, $V_{y}$ is the effect of the constriction and $V$ is the time-dependent bias potential. The scattering potential is assumed to be nonzero only in a small interval around the center of the constriction while $V_{y}$ grows to infinity with $|y| \rightarrow \infty$. We have the total current operator in $x$ direction

$$
\begin{aligned}
& \hat{I}(x)=-\frac{i \hbar e}{2 m} \int d y \\
& \sum_{\sigma}\left[\hat{\psi}_{\sigma}^{\dagger}(x, y) \partial_{x} \hat{\psi}_{\sigma}(x, y)-\left(\partial_{x} \hat{\psi}_{\sigma}^{\dagger}(x, y)\right) \hat{\psi}_{\sigma}(x, y)\right] .
\end{aligned}
$$

In absence of a bias voltage, the Hamiltonian can be diagonalized in the space of scattering states described by fermionic operators $\hat{\psi}_{k, \bar{n}}(x)$ with the channel quantum number including the electron spin $\bar{n}=(n, \sigma)$. Far from the region $V_{x} \neq 0$, we have

$$
\begin{aligned}
\hat{\psi}_{\sigma}(x, y)= & \int \frac{d k}{\sqrt{2 \pi}} \psi_{n}(y)\left[\left(e^{i k x}+\tilde{r}_{n} e^{-i k x}\right) \theta(-k x)\right. \\
& \left.+\tilde{t}_{n} e^{i k x} \theta(k x)\right] \hat{\psi}_{k, \bar{n}}
\end{aligned}
$$

Here we distinguish between the normalized transversal mode wavefunction $\psi_{n}$ and the mode operator $\hat{\psi}_{k, \bar{n}}, k$ is the longitudinal wave vector, $\tilde{r}_{n}$ and $\tilde{t}_{n}$ are reflection and transmission scattering amplitudes, respectively. Due to unitarity, transmission $T_{n}=\left|\tilde{t}_{n}\right|^{2}$ and reflection coefficient $R_{n}=\left|\tilde{r}_{n}\right|^{2}$ satisfy $R_{n}+T_{n}=1$. We assume that the junction is long enough to treat $k$ as a continuous parameter. The transverse mode index $n$ is kept as discrete. The energy has the structure $E=E_{n}+\hbar^{2} k^{2} / 2 m$, where $E_{n}$ is for the transversal part.

\section{A. Expansion around Fermi level}

We are interested only in phenomena in a narrow part of the electron band around Fermi level $E_{F}: \underline{\underline{4}}$ In particular, we assume $k_{B} T, e V, \hbar|\omega| \ll E_{F}$. We also neglect the exact structure of wavefunctions in the region $V_{x} \neq 0$ and take only asymptotic states, like in (5). We denote Fermi wave numbers $k_{n}=\sqrt{2 m\left(E_{F}-E_{n}\right)} / \hbar$ and Fermi velocities $v_{n}=\hbar k_{n} / m$. Only modes with $E_{n}<E_{F}$ contribute at zero temperature.

We construct an extended Hilbert space consisting of left and right going states, $L$ and $R$, respectively. The relation between operators in the reduced and standard space is

$$
\begin{aligned}
& \hat{\psi}_{L \bar{n}}(x)=\int_{k<0} \frac{d k d x^{\prime} d y}{2 \pi} e^{i k\left(x^{\prime}-x\right)} \psi_{n}^{*}(y) \hat{\psi}_{\sigma}\left(x^{\prime}, y\right), \\
& \hat{\psi}_{R \bar{n}}(x)=\int_{k>0} \frac{d k d x^{\prime} d y}{2 \pi} e^{i k\left(x^{\prime}-x\right)} \psi_{n}^{*}(y) \hat{\psi}_{\sigma}\left(x^{\prime}, y\right) .
\end{aligned}
$$

In fact, only $k \sim-k_{n}$ and $k \sim k_{n}$ play a role for $L$ and $R$, respectively. The actual dynamics of states deep below or above the Fermi sea can be ignored and its only reminder will be some ultraviolet regularization or cutoff. The new operators satisfy anticommutation relations

$$
\begin{aligned}
& \left\{\hat{\psi}_{A \bar{n}}(x), \hat{\psi}_{B \bar{m}}\left(x^{\prime}\right)\right\}=0, \\
& \left\{\hat{\psi}_{A \bar{n}}(x), \hat{\psi}_{B \bar{m}}^{\dagger}\left(x^{\prime}\right)\right\}=\delta_{A B} \delta_{\bar{n} \bar{m}} \delta\left(x-x^{\prime}\right)
\end{aligned}
$$

for $A, B=L, R$.

With help of the above stated approximations, we can write $\hat{H}$ in the new space as

$$
\begin{aligned}
& \hat{H}_{0}+\sum_{\bar{n}} q_{n} v_{n}\left[\hat{\psi}_{L \bar{n}}^{\dagger}(0) \hat{\psi}_{R \bar{n}}(0)+\hat{\psi}_{R \bar{n}}^{\dagger}(0) \hat{\psi}_{L \bar{n}}(0)\right]+(8) \\
& \sum_{\bar{n}} \int d x e V(x, t)\left[\hat{\psi}_{L \bar{n}}^{\dagger}(x) \hat{\psi}_{L \bar{n}}(x)+\hat{\psi}_{R \bar{n}}^{\dagger}(x) \hat{\psi}_{R \bar{n}}(x)\right],
\end{aligned}
$$

where $\hat{H}_{0}$ is equal to

$$
\sum_{\bar{n}} \int d x i \hbar v_{n}\left[\hat{\psi}_{L \bar{n}}^{\dagger}(x) \partial_{x} \hat{\psi}_{L \bar{n}}(x)-\hat{\psi}_{R \bar{n}}^{\dagger}(x) \partial_{x} \hat{\psi}_{R \bar{n}}(x)\right] .
$$

It is necessary to regularize the second term in Eq. (8) to define the transmission and the reflection coefficients, $T_{n}=\cos ^{2}\left(q_{n} / \hbar\right)$ and $R_{n}=\sin ^{2}\left(q_{n} / \hbar\right)$, respectively (see the discussion in the appendix $A)$.

The current operator (5) in the new basis is replaced by

$$
\hat{I}(x)=\sum_{\bar{n}} e v_{n}\left(\hat{\psi}_{R \bar{n}}^{\dagger}(x) \hat{\psi}_{R \bar{n}}(x)-\hat{\psi}_{L \bar{n}}^{\dagger}(x) \hat{\psi}_{L \bar{n}}(x)\right) .
$$

As we will later see, it is disadvantegous to operate directly with the field operators. To circumvent this it is possible to introduce bosonic operators (viz. quadratic forms of Fermion operators) and to develop a closed algebra for those. Since current operators are generally non-commuting, we have to cope with their algebra. To this end, we introduce the following auxiliary operators

$$
\begin{aligned}
& \hat{I}_{0 \bar{n}}\left(x_{n}\right)=\frac{e v_{n}}{2}\left(\hat{\psi}_{L \bar{n}}^{\dagger}(x) \hat{\psi}_{L \bar{n}}(x)+\hat{\psi}_{R \bar{n}}^{\dagger}(-x) \hat{\psi}_{R \bar{n}}(-x)\right), \\
& \hat{I}_{1 \bar{n}}\left(x_{n}\right)=\frac{e v_{n}}{2}\left(\hat{\psi}_{L \bar{n}}^{\dagger}(x) \hat{\psi}_{L \bar{n}}(x)-\hat{\psi}_{R \bar{n}}^{\dagger}(-x) \hat{\psi}_{R \bar{n}}(-x)\right), \\
& \hat{I}_{2 \bar{n}}\left(x_{n}\right)=\frac{i e v_{n}}{2} \hat{\psi}_{L \bar{n}}^{\dagger}(x) \hat{\psi}_{R \bar{n}}(-x)+\text { h.c. }, \\
& \hat{I}_{3 \bar{n}}\left(x_{n}\right)=\frac{e v_{n}}{2} \hat{\psi}_{L \bar{n}}^{\dagger}(x) \hat{\psi}_{R \bar{n}}(-x)+\text { h.c. }
\end{aligned}
$$


with $x_{n}=x / v_{n}$. Note that $x_{n}$ has the unit of time. It will be more convenient for us than length units. The Hamiltonian (8) can be now written as

$$
\begin{aligned}
\hat{H} & =\hat{H}_{0}+\sum_{\bar{n}} 2 q_{n} \hat{I}_{3 \bar{n}}(0) / e \\
& +\sum_{\bar{n}, \pm} \int d s V\left( \pm s v_{n}, t\right)\left[\hat{I}_{0 \bar{n}}(s) \pm \hat{I}_{1 \bar{n}}(s)\right]
\end{aligned}
$$

The current takes the form

$$
\hat{I}(x)=\sum_{\bar{n}}\left[\hat{I}_{0 \bar{n}}\left(-x_{n}\right)-\hat{I}_{0 \bar{n}}\left(x_{n}\right)-\hat{I}_{1 \bar{n}}\left(-x_{n}\right)-\hat{I}_{1 \bar{n}}\left(x_{n}\right)\right] \text {. }
$$

Using arguments similar to bosonization ${ }^{30}$ (see appendix A), we get the following commutation rule

$$
\begin{aligned}
& {\left[\hat{I}_{j \bar{n}}(s), \hat{I}_{k \bar{m}}\left(s^{\prime}\right)\right]=i e^{2} \delta_{\bar{n} \bar{m}} \delta_{j k} \partial_{s} \delta\left(s-s^{\prime}\right) / 4 \pi} \\
& +\sum_{l} i \epsilon_{j k l} e \delta_{\bar{n} \bar{m}} \hat{I}_{l \bar{n}}(s) \delta\left(s-s^{\prime}\right)
\end{aligned}
$$

with $\epsilon_{j k l}$ equal +1 for $j k l=123,231,312,-1$ for $j k l=$ $321,213,132$ and zero otherwise. The last useful commutator is

$$
\left[\hat{H}_{0}, \hat{I}_{j \bar{n}}(s)\right]=-i \hbar \partial_{s} \hat{I}_{j \bar{n}}(s)
$$

and one can in principle write

$$
\hat{H}_{0}=2 \pi \hbar \int d s \sum_{j \bar{n}} \hat{I}_{j \bar{n}}^{2}(s) .
$$

The current $\hat{I}_{0}$ corresponds to the total charge changes in the leads. It is preserved due to absence of capacitive effects and behaves like a free bosonic field. Without the scattering term $\hat{I}_{3}$ in (12) only the first term in (14) needs to be considered in the dynamics, since only $\hat{I}_{0}$ and $\hat{I}_{1}$ are dynamical variables. The system could be fully bosonized and all correlation functions can be calculated. A nonvanishing scattering retains some fermionic features due to the commutator $\left[\hat{I}_{1}, \hat{I}_{3}\right]$. As a consequence the fluctuations become non-Gaussian.

\section{B. Equilibrium averages}

Far from the junction and without bias potential, we can write the initial density matrix as

$$
\hat{\rho}=\exp \left(-\hat{H}_{0} / k_{B} T\right) / \operatorname{Tr} \exp \left(-\hat{H}_{0} / k_{B} T\right) .
$$

The commutator (15) gives the useful relation

$$
\operatorname{Tr} \hat{\rho} \hat{I}_{j \bar{n}}(s) \hat{A}=\operatorname{Tr} \hat{\rho} \hat{A} \hat{I}_{j \bar{n}}\left(s+i \hbar / k_{B} T\right) .
$$

A similar relation has been introduced in Ref. 32. For convenience we rewrite the previous equation and the relations (14) in the frequency domain for the operators

$$
\hat{I}_{j \bar{n}}(\omega)=\int d s e^{i \omega s} \hat{I}_{j \bar{n}}(t) .
$$

From now on, a Greek argument will always denote the Fourier-transformed operators. The current algebra reads now

$$
\begin{aligned}
& {\left[\hat{I}_{j \bar{n}}(\alpha), \hat{I}_{k \bar{m}}(\beta)\right]=e^{2} \delta_{\bar{n} \bar{m}} \delta_{j k} \alpha \delta(\alpha+\beta) / 2} \\
& +\sum_{l} i \epsilon_{j k l} e \delta_{\bar{n} \bar{m}} \hat{I}_{l \bar{n}}(\alpha+\beta)
\end{aligned}
$$

Eq. (18) reproduces the fluctuation-dissipation theorem ${ }^{33}$

$$
\operatorname{Tr} \hat{\rho} \hat{I}_{j \bar{n}}(\omega) \hat{A}=e^{\frac{\hbar \omega}{k^{T} T}} \operatorname{Tr} \hat{\rho} \hat{A} \hat{I}_{j \bar{n}}(\omega)
$$

With the above derived tools it is now straightforward to calculate equilibrium averages. As examples we obtain

$$
\begin{aligned}
& \operatorname{Tr} \hat{\rho} \hat{I}_{j \bar{n}}(\omega)=0, \\
& \operatorname{Tr} \hat{\rho} \hat{I}_{j \bar{n}}(\alpha) \hat{I}_{k \bar{m}}(\beta)=\frac{e^{2} \delta_{\bar{n} \bar{m}} \delta_{j k}}{4} \delta(\alpha+\beta)(w(\alpha)+\alpha), \\
& \operatorname{Tr} \hat{\rho} \hat{I}_{j \bar{n}}(\alpha) \hat{I}_{k \bar{m}}(\beta) \hat{I}_{l \bar{p}}(\gamma)=\frac{i e^{3} \epsilon_{j k l} \delta_{\bar{n} \bar{m}} \delta_{\bar{n} \bar{p}}}{8} \delta(\alpha+\beta+\gamma) \\
& {[u(\beta)(w(\alpha)-w(\gamma))+w(\alpha)-w(\beta)+w(\gamma)+\alpha-\gamma],}
\end{aligned}
$$

where we introduced

$$
\begin{aligned}
u(\omega) & =\operatorname{coth}\left(\hbar \omega / 2 k_{B} T\right) \\
w(\omega) & =\omega u(\omega) \\
w(s) & =-\lim _{\epsilon \rightarrow 0} \operatorname{Re} \frac{\pi\left(k_{B} T / \hbar\right)^{2}}{\sinh ^{2}\left(\pi s k_{B} T / \hbar+i \epsilon\right)} .
\end{aligned}
$$

We note that $w(s=0)$ is singular and all practical calculations need a proper regularization around $s=0$. This can for example be an ultraviolet cutoff of the order of the Fermi energy in the frequency domain.

\section{Heisenberg equations}

To generalize the previous method to a nonequilibrium situation, it is necessary to solve the Heisenberg equations for the various current operators we have introduced. It is a major advantage of the current algebra, we have introduced, that this can be done exactly. From now on, we switch to Heisenberg picture, so all operators will be transformed as

$$
i \hbar \partial_{t} \hat{A}(t)=[\hat{A}(t), \hat{H}(t)]+i \hbar \tilde{\partial}_{t} \hat{A}(t)
$$

Here the $\tilde{\partial}_{t}$ denotes explicit time dependence of the operator. Remarkable, the Heisenberg equations can be solved completely for the operators $\hat{I}_{j \bar{n}}$. The details are described in the appendix B and in the following we merely present the results. To distinguish between time and spatial coordinate (which has the same unit, when divided by Fermi velocity) we will denote by $\hat{A}(s, t)$ an operator at position $s$ and time $t$. We assume an arbitrarily time-dependent bias potential $V(x, t)=\theta(-x) V(t)$ and neglect some regularization issues around $x=0$ and 
$x \rightarrow-\infty$, which can of course be properly handled using the procedures mentioned previously. We find for the Heisenberg operators

$$
\begin{aligned}
& \hat{I}_{0 \bar{n}}(s, t)=\hat{I}_{0 \bar{n}}(t+s) \\
& \hat{I}_{1 \bar{n}}(s, t)=\hat{I}_{1 \bar{n}}(t+s) \text { for } s>0 \\
& \hat{I}_{1 \bar{n}}(s, t)=\left(T_{n}-R_{n}\right) \hat{I}_{1 \bar{n}}(t+s) \\
& -\frac{e^{2}}{2 \pi \hbar} T_{n} V(t+s)-2 \sqrt{R_{n} T_{n}} \hat{J}_{\bar{n}}^{\Phi}(t+s) \text { for } s<0
\end{aligned}
$$

with

$$
\hat{J}_{\bar{n}}^{\Phi}(s)=\hat{I}_{2 \bar{n}}(s) \cos \Phi(s)-\hat{I}_{3 \bar{n}}(s) \sin \Phi(s) .
$$

and

$$
\Phi(s)=-\int_{-\infty}^{s} d t e V(t) / \hbar
$$

Similar expressions can be obtained for $\hat{I}_{2 \bar{n}}(s, t)$ and $\hat{I}_{3 \bar{n}}(s, t)$ and are presented in the appendix B. The big advantage of the above equations is that the Heisenberg current operators are represented by linear combinations of equilibrium operators. Hence, their averages are straightforwardly obtained using the results of the previous subsection.

We conclude this section by emphasizing the main results we have obtained so far. The calculation of higher order cumulants of the current in a mesoscopic junction using the standard scattering approach is quite a demanding task. This is true in particular at finite frequency, when operator ordering issues become even more prominent. The method, we have developed in this section builds in an elegant way on a very simple algebra of current operators (interestingly resembling the usual angular momentum algebra). Our method allows to obtain in a relatively straightforward manner arbitrarily ordered current cumulants of (almost) any order.

\section{THE MEASUREMENT - POVM}

We now turn to the main topic of the article, how the current correlators at high frequencies can be measured and how the measurement protocol itself influences the measurement. We will first treat the measurement on a phenomenological basis using the so-called positive operator-valued measure (POVM). In this formalism, the measurement is described by Kraus operators, $\stackrel{20}{=}$ which we phenomenologically assume to have the form

$$
\hat{K}[I]=\int D \phi \mathcal{T} e^{\int d t\left[i \phi(t)\left(\hat{I}_{R}(t)-I(t)\right) / e-\phi^{2}(t) / \tau\right]} .
$$

Here $\mathcal{T}$ denotes time ordering and

$$
\hat{I}_{R}(t)=\int d x \hat{I}(x, t) g\left(x-x_{R}\right)
$$

is current operator averaged over a spatial region near the point $x_{R}>0$. By virtue of Naimark's theorem ${ }^{22}$, the completeness relation $\int D I \hat{K}^{\dagger}[I] \hat{K}[I]=1$ is sufficient to guarantee that the measurement corresponds to a usual projective measurement in some extended Hilbert space including the detector.

Additionally, we assume as usual that the detector and the system are initially uncorrelated ${ }^{23}$ The spatial resolution is taken into account by a convolution function $g(x)=e^{-x^{2} / 2 \Delta x^{2}} / \sqrt{2 \pi} \Delta x$, which is parametrized by the spatial sensitivity $\Delta x$. To ensure, that the current on one side of the junction is measured, we also take $\Delta x \ll x_{R}$. The Kraus operator contains the parameter $\tau$, which plays the role of a measurement sensitivity. Varying $\tau$, the measurement changes from a weak, but nondemolishing measurement in the limit $\tau \rightarrow 0$ to a strong projective measurement for $\tau \rightarrow \infty$, which however yields not the expected result due to a large disturbing noise of the detector. For practical reasons, we convert distances into times, namely, $\tau_{n}=\Delta x / v_{n}$ and $t_{n}=x_{R} / v_{n}$. The physical meaning of $\tau_{n}$ is therefore the time, which an electron in the lead in channel $n$ effectively interacts with the detector. On the other hand, $t_{n}$ is related to the time-of-flight between the scatterer and the detector.

The probability density of a given time trace for the current is given by a $\mathrm{POVM}^{21}$

$$
\rho[I]=\operatorname{Tr} \hat{\rho} \hat{K}^{\dagger}[I] \hat{K}[I],
$$

The generating functional is defined as

$$
\mathcal{S}[\chi]=\ln \left\langle\exp \left[i \int d t \chi(t) I(t) / e\right]\right\rangle_{\rho}
$$

where the average is defined as

$$
\langle\ldots\rangle_{\rho}=\int D I \rho[I] \ldots
$$

Using the Kraus operators, one obtains for the generating functional

$$
\mathcal{S}[\chi]=\ln \int D \phi e^{S[\chi, \phi]-\int d t\left(2 \phi^{2}(t)+\chi^{2}(t) / 2\right) / \tau},
$$

where $S[\chi, \phi]$ is the standard Keldysh functional, defined as

$$
\begin{aligned}
S[\chi, \phi]= & \ln \operatorname{Tr}\left\{\hat{\rho} \tilde{\mathcal{T}} \exp \left[\int \frac{i d t}{2 e}(\chi(t)+2 \phi(t)) \hat{I}_{R}(t)\right]\right. \\
& \left.\times \mathcal{T} \exp \left[\int \frac{i d t}{2 e}(\chi(t)-2 \phi(t)) \hat{I}_{R}(t)\right]\right\} .
\end{aligned}
$$

The measure $D \phi$ is scaled to keep $\mathcal{S}[\chi \equiv 0]=0$.

To calculate averages we need the transformations presented in Appendix C. For future convenience we shall denote $s_{ \pm n}=s \pm t_{n}$ and

$$
\theta_{n}(s)=\theta(s) h_{n}^{\prime}(s), h_{n}(s)=e^{-\frac{s^{2}}{4 \tau_{n}^{2}}} / 2 \sqrt{\pi} \tau_{n} .
$$


The mean current is

$$
\langle I(t)\rangle_{\rho}=-\left.i e \frac{\delta \mathcal{S}}{\delta \chi(t)}\right|_{\chi \equiv 0}=\frac{e^{2}}{\pi \hbar} \sum_{n} V\left(t_{-n}\right) T_{n} .
$$

Hence, the conductance is robust against detector backaction as it does not depend on $\tau$. In the limit of no-delay measurement $\left(t_{n} \rightarrow 0\right)$, the measured current follows the (time-dependent) voltage

$$
\langle I(t)\rangle_{\rho}=G V(t), G=\frac{2 e^{2}}{h} \sum_{n} T_{n} .
$$

The correlation function is

$$
\langle\delta I(a) \delta I(b)\rangle_{\rho}=\left.\frac{-e^{2} \delta^{2} \mathcal{S}}{\delta \chi(a) \delta \chi(b)}\right|_{\chi \equiv 0}=e^{2} P(a, b)
$$

for $\delta I=I-\langle I\rangle_{\rho}$. We are interested in frequency scales of the current fluctuations in the regime $\omega \ll \tau_{n}^{-1}$. Note, that the scale $1 / \tau_{n}$ plays a role of the maximal bandwidth of the detector. This means that the detector instantaneously measures the current, which is a reasonable assumption also in typical experiments.

In this case (see details in the appendix $\mathrm{C}$ ) the noise measured at the detector contains several contributions and can be written as

$$
P(a, b)=\frac{1}{\tau} \delta(a-b)+P_{\tau}(a-b)+P_{0}(a-b)+P_{e}(a, b) .
$$

The first term is just a Gaussian white noise of the detector, which one would also expect classically. The Fourier transforms of the second term can be written as

$$
\begin{aligned}
& P_{\tau}(\omega)=\frac{\tau}{4 \pi^{2}}\left|\sum_{n}\left(2 i \theta_{n}(\omega)+\omega R_{n} e^{2 i \omega t_{n}}\right)\right|^{2}, \\
& \theta_{n}(\omega) \simeq-h_{n}(0)-i \omega / 2
\end{aligned}
$$

with $\theta_{n}$ defined by (35). Therefore, $P_{\tau}$ has a frequency dispersion solely determined by the properties of the detector. It is independent of the bias voltage and can, thus, add an unknown contribution to the voltage independent background noise. Note, that it can be also made negligible by a suitable choice of the detector parameters,

The third term has the form

$$
P_{0}(\omega)=\frac{w(\omega)}{\pi} \sum_{n}\left(T_{n}^{2}+R_{n}\left(1-\cos \left(2 \omega t_{n}\right)\right) .\right.
$$

Here $w(\omega)$ is defined by Eq. (23). This term is also independent of the bias voltage and can be neglected at sufficiently low temperatures. .

Finally the most interesting contribution to the noise is the voltage-dependent part $P_{e}$, which we can write with the help of (C6) and $P_{e}(a, b)=\sum_{n} P_{n}(a, b)$ as

$$
P_{n}(a, b)=R_{n} T_{n} \frac{w(a-b)}{\pi \Gamma_{n}(a-b)} \cos \varphi\left(a_{-n}, b_{-n}\right)
$$

with $\Gamma_{n}(t)=\exp \left(\tau\left(h_{n}(0)-h_{n}(t)\right) / 4\right)$ and

$$
\varphi(a, b)=\Phi(a)-\Phi(b)=\int_{a}^{b} d t e V(t) / \hbar .
$$

Still, this result can be applied to arbitrary frequency after multiplying $P_{n}\left(\omega, \omega^{\prime}\right)$ by the damping factor $e^{-\left(\omega^{2}+\omega^{\prime 2}\right) \tau_{n}^{2} / 2}$. The noise (42) contains the usual symmetrized quantum noise in agreement with existing results. .34 The factor $\Gamma$ results from the detector backaction on the current during the measurement on a timescale set by the phenomenological parameter $\tau$. Note that $\Gamma(t \rightarrow 0) \rightarrow 1$. A high voltage $V$ leads to a strongly oscillating term $\cos (\varphi)$, so that $P_{n}$ effectively probes the short time-scale $t \sim \hbar / \mathrm{eV}$. Hence, in the limit of high voltage we can take $\Gamma(\hbar / \mathrm{eV}) \rightarrow 1$ and the noise is independent of the detector's backaction. To illustrate this effect more clearly let us take $V=$ const and $t_{n}=0$. In this case

$$
\begin{aligned}
P_{n}\left(\omega, \omega^{\prime}\right) & =2 \pi \delta\left(\omega+\omega^{\prime}\right) P_{n}(\omega), \\
P_{n}(t) & =R_{n} T_{n} \frac{w(t)}{\pi \Gamma_{n}(t)} \cos (e V t / \hbar) .
\end{aligned}
$$

In Fourier space we obtain the convolution

$$
\begin{aligned}
P_{n}(\omega)= & \int \frac{d \alpha}{(2 \pi)^{2}} R_{n} T_{n} \Gamma_{n}^{-1}(\omega-\alpha) \\
& \times[w(\alpha+e V / \hbar)+w(\alpha-e V / \hbar)] .
\end{aligned}
$$

Due to the symmetry of $\Gamma(\omega)=\Gamma(-\omega)$ and the fact that $\Gamma_{n}^{-1}(\omega \rightarrow \infty)$ vanishes, we can replace $\Gamma_{n}^{-1}(\omega) \rightarrow$ $2 \pi \delta(\omega)$ at high voltage and get the shot noise $P_{n}=$ $R_{n} T_{n}|e V| / \pi \hbar$. Let us find the backaction corrections to the shot noise at zero frequency $(\omega=0)$,

$$
P_{n}(0)=\int \frac{d \alpha}{2 \pi^{2}} R_{n} T_{n} w(\alpha) \Gamma_{n}^{-1}(\alpha+e V / \hbar)
$$

For $\tau \ll \tau_{n}$, have

$$
\Gamma_{n}^{-1}(\alpha)=\left(2 \pi-\sqrt{\pi} \tau / 4 \tau_{n}\right) \delta(\alpha)+\tau e^{-\alpha^{2} \tau_{n}^{2}} / 4
$$

and finally at zero temperature $(w(\alpha)=|\alpha|)$ we get

$$
P_{n}(0)=R_{n} T_{n}\left[|e V| / \pi \hbar+q\left(|e V| \tau_{n} / \hbar\right) \tau / 8 \pi^{2} \tau_{n}^{2}\right]
$$

with $q(x)=e^{-x^{2}}-2 x \int_{x}^{\infty} d z e^{-z^{2}}$.

To illustrate the effect of the backaction due to the detection process we show in Fig. 1 the low- and the high-frequency noise for different detector parameters $\tau$. The upper panel shows that the effect of the detector is strongest for small voltages $e V \lesssim \hbar / \tau_{n}$. We can interpret this as follows: The additional noise added by the detection is dominant as long as the electrons flow with a rate smaller than the inverse interaction time with the detector $\lesssim 1 / \tau_{n}$ through the contact. The influence of the backaction becomes negligible if the electrons flow at a higher rate, so that they do not feel the backaction and 

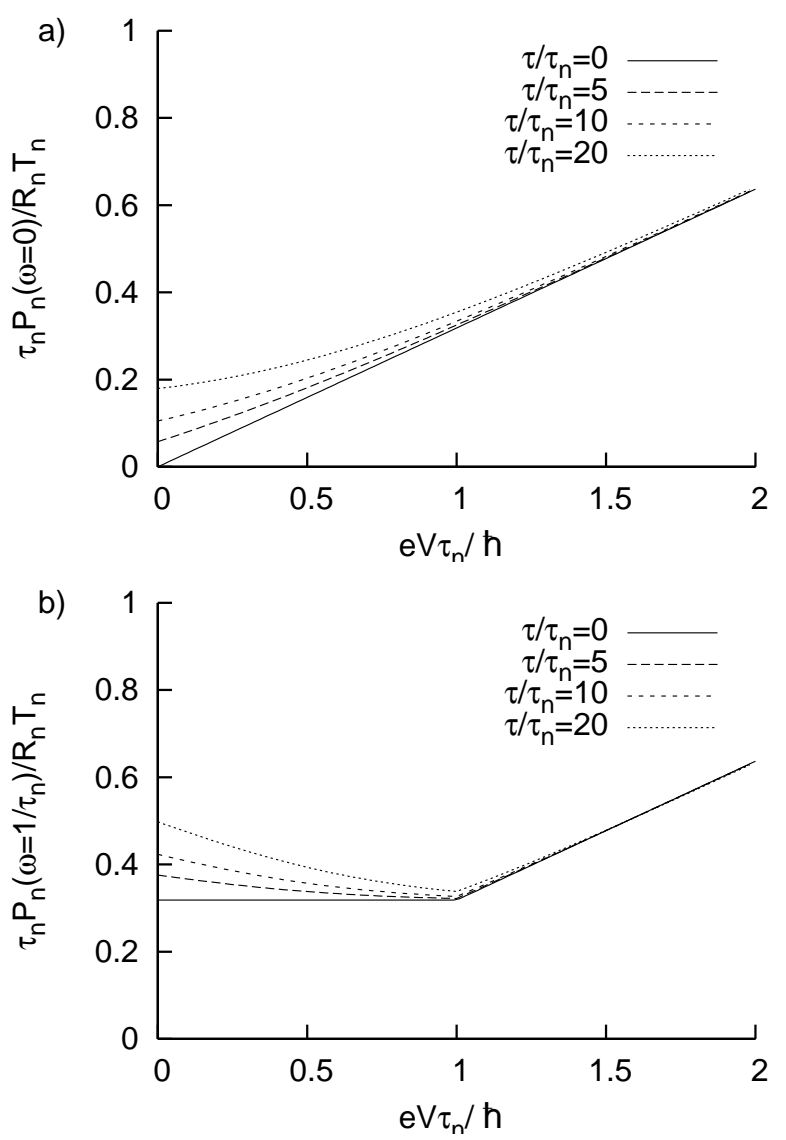

FIG. 1: The voltage-dependent noise $P_{n}$ at dc voltage and zero temperature for zero frequency $\omega=0$ (a) and a finite frequency $\omega=1 / \tau_{n}$ (b). In both cases the additional noise due to the backaction of the detector leads to drastic changes in the voltage dependence for $e V \lesssim 1 / \tau_{n}$. The magnitude of the noise increase depends on the ratio $\tau / \tau_{n}$. For larger voltages the usual shot noise is recovered.

the full shot noise is recovered. A similar picture holds if the detector is sensitive to the finite-frequency current correlations. The signature of the quantum transport at $\hbar \omega=e V$ is gradually smeared out if the detector becomes slower, viz. $\tau \gtrsim \tau_{n}$. Interestingly, the noise first decreases with a finite voltage. Finally we note, that Fig. 1 shows numerically that the correction is small even at $\tau \sim \tau_{n}$.

\section{A. Many detectors}

We can generalize the Kraus operator (28) to the case of many detectors

$$
\hat{K}[I]=\int D \phi \mathcal{T} e^{\int d t \sum_{A}\left(i \phi_{A}(t)\left(\hat{I}_{A}(t)-I_{A}(t)\right) / e-\phi_{A}^{2}(t) / \tau_{A}\right)}
$$

In particular, we take two independent detectors placed on the left and right hand side of the QPC. They are described similarly as in the single detector case, namely

$$
\hat{I}_{A}(t)=\int d x \hat{I}(x, t) g_{A}\left(x-x_{A}\right), \text { for } A=L, R .
$$

Here we assumed $x_{R}>0$ and $x_{L}<0$ and $g_{A}(x)=$ $e^{-x^{2} / 2 \Delta x_{A}^{2}} / \sqrt{2 \pi} \Delta x_{A}$ with $\Delta x_{A} \ll\left|x_{A}\right|$. The latter assumption means that the measuring regions of the two detectors do not overlap. We also denote the interaction time of the detector with the electrons as $\tau_{A n}=\Delta x_{A} / v_{n}$.

The conductance (37) remains unchanged but the noise can be now measured in four different ways. Namely, we can define the four correlators

$$
\left\langle\delta I_{A}(a) \delta I_{B}(b)\right\rangle_{\rho}=-\left.\frac{e^{2} \delta \mathcal{S}}{\delta \chi_{A}(a) \delta \chi_{B}(b)}\right|_{\chi \equiv 0}=e^{2} P_{A B}(a, b)
$$

with $A, B=R, L$.

We shall consider only frequency scales $\omega \ll v_{n} /\left|x_{A}\right|$, so that we can use $t_{n}=0$ in all derivations. Then, similarly as in the previous section (details in the appendix C), we obtain expressions for all correlators of the form

$$
\begin{aligned}
P_{A B}(a, b)= & \frac{1}{\tau_{A}} \delta_{A B} \delta(a-b)+P_{A B}(a-b) \\
& +P_{0}(a-b)+P_{e}(a, b) .
\end{aligned}
$$

Here, $P_{0}$ is defined by Eq. (41) with $t_{n}=0$. The excess noise $P_{e}$ is defined by Eq. (42), but with

$$
\Gamma_{n}(t)=\exp \left(\sum_{A} \tau_{A}\left(h_{A n}(0)-h_{A n}(t)\right) / 4\right) .
$$

Note that the excess contribution is the same for all correlators and depends on the parameters of both detectors. The voltage independent contributions differ for the auto-correlators and the cross-correlators. We have

$$
P_{A A}(\omega)=P_{\tau A}(\omega)+\frac{\omega^{2} \tau_{B}}{4 \pi^{2}}\left(\sum_{n} T_{n}\right)^{2}
$$

where $P_{\tau A}$ is defined by (40) with $\tau=\tau_{A}$ and $\tau_{n}=\tau_{A n}$ and $B=L, R$ for $A=R, L$, respectively. Finally, for $A \neq B$ we find

$$
\begin{aligned}
& P_{A B}(\omega)=\frac{\omega}{4 \pi^{2}} \sum_{n} T_{n} \sum_{m} \\
& {\left[\tau_{A}\left(2 i \theta_{A m}(\omega)-R_{m} \omega\right)+\tau_{B}\left(-2 i \theta_{B m}^{*}(\omega)-R_{m} \omega\right)\right] .}
\end{aligned}
$$

The possibility to measure several independent correlators has interesting consequences. In the case of a single detector one could in practice measure only the voltagedependent contribution of the noise as the offset noise has generally an unknown value and is subtracted. However, the use of two independent detectors helps to estimate the background noise in the auto-correlation signal. Hence, comparing cross- and auto-correlations we also can get a rough estimate of the offset noise. Furthermore, in the limit $\tau_{L, R} \rightarrow 0$ we get $P_{L R}=P_{0}+P_{e}$. As $P_{e}$ is independently known from the auto-correlation measurement, we can get information about the voltageindependent part of the noise $P_{0}$. 


\section{B. Higher cumulants}

Now we consider the effect of our detection scheme on the third and fourth cumulants of the current fluctuations. While these are harder to measure than noise correlations, they contain a tremendous deeper information that the current. It should be noted, that the third cumulant already at zero frequency contains a non-trivial ordering of the current operators ${ }^{6}$ and we can expect a similar non-trivial effect of the detection scheme. Quite generally the third and forth cumulants are defined as

$$
\begin{aligned}
\langle\langle A B C\rangle\rangle= & \langle\delta A \delta B \delta C\rangle, \\
\langle\langle A B C D\rangle\rangle= & \langle\delta A \delta B \delta C \delta D\rangle-\langle\delta A \delta B\rangle\langle\delta C \delta D\rangle \\
& -\langle\delta A \delta C\rangle\langle\delta B \delta D\rangle-\langle\delta A \delta D\rangle\langle\delta C \delta B\rangle .
\end{aligned}
$$

Here $\delta X=X-\langle X\rangle$ are fluctuations of some observable. In the following these will be current fluctuations operators at different times.

We now apply out model of a detector, which is parametrized by a Kraus operator to calculate the cumulants. Unfortunately, the general expressions are too cumbersome to be shown here and we discuss only a limiting case below. Hence, we take the limit $\left|\tau_{n} \omega\right|, \tau / \tau_{n} \ll 1$, where $\omega$ describes relevant frequency scale of the measurement. In this case the only effect of the detector is a large white Gaussian offset noise $1 / \tau$. It only adds as a constant to the second cumulant, while higher cumulants are unaffected.

The derivation of the third cumulant is given in the appendix D. The result for $C_{3}(a, b, c)=\langle\langle I(a) I(b) I(c)\rangle\rangle$ is

$$
\begin{aligned}
& C_{3}(a, b, c)=e^{3} \sum_{n}\left\{R_{n} T_{n}\left(R_{n}-T_{n}\right) \times\right. \\
& \frac{e V\left(a_{-n}\right)}{\pi \hbar} \delta(a-b) \delta(a-c)+\sum_{\sigma(a b c)} \int d s P_{n}(a, b ; s) \times \\
& \left.\left[\left(2 T_{n}-1\right) w\left(s-c_{-n}\right)+w\left(s-c_{+n}\right)\right] \pi / 2\right\}
\end{aligned}
$$

where the summation is over all permutations of the set $a b c$. At zero flight time and either zero temperature and frequency or tunneling limit only the first term survives. The last part contains the so-called noise susceptibility 14

$$
P_{n}(a, b ; s)=\frac{\hbar \delta P_{n}(a, b)[V]}{e \delta V(s)},
$$

generalized here to an arbitrary time-dependence of the bias. The result (57) in the limit of zero flight time has a somewhat simpler form than the existing results. ${ }^{24}$ The identification of the noise susceptibility in the third cumulant has important consequences on its detection. A general detector, which is equivalent to an electromagnetic environment, and weakly coupled here, gives cor- rections to (57) of the form ${ }^{8-10,27}$

$$
\begin{aligned}
& C_{3}^{e n}\left(\omega_{1}, \omega_{2}, \omega_{3}\right)=\sum_{\sigma(i j k)} \int d \omega P\left(\omega_{i}, \omega_{j} ; \omega\right) \times \\
& {\left[b_{0}(\omega) w(\omega) \delta\left(\omega-\omega_{k}\right)+b_{1}(\omega) P\left(-\omega, \omega_{k}\right) / e^{2}\right] / 4 .}
\end{aligned}
$$

Here $P(\alpha, \beta ; s)=\delta P(\alpha, \beta) / \delta V(s)$ is the noise susceptibility. The function $b_{0}$ represents the influence of environmental noise and $b_{1}$ is the voltage-dependent feedback of the environment due to the noise of the system. They can in principle be modeled by an effective electric circuit. In practice, the precise environmental circuit is not known, and the functions are determined by fitting some model environment. 27

An important case in practice is a constant bias voltage, $V=$ const. The expressions for the noise susceptibility and the third cumulant are simplified considerably. The noise susceptibility turns out to be

$$
\begin{aligned}
P_{n}(\alpha, \beta ; \omega)= & R_{n} T_{n} \delta(\alpha+\beta+\omega) e^{-i \omega t_{n}} \times \\
& \sum_{\gamma=\alpha, \beta}[w(\gamma-e V / \hbar)-w(\gamma+e V / \hbar)] / \omega
\end{aligned}
$$

The noise susceptibility is shown in Fig. 2 for different temperatures. In all cases, it preserves the symmetry $\alpha \leftrightarrow \pm \beta$. At zero temperature, a non-analyticity occurs along lines $|e V / \hbar|=|\alpha|,|\beta|$, which is smeared out at increasing temperatures.

The third cumulant also becomes much simpler. Due to the relation $C_{3}(\alpha, \beta, \omega)=2 \pi \delta(\alpha+\beta+\omega) \bar{C}_{3}(\alpha, \beta)$, it effectively depends only on the frequencies $\alpha$ and $\beta$. The delta function imposes the constraint $\alpha+\beta+\omega=$ 0 so only two of the three frequencies are independent. The cumulant is hence symmetric under changes $\alpha, \beta \leftrightarrow$ $\omega=-\alpha-\beta$. In the following expressions, we either use two independent arguments $(\alpha, \beta)$ or three constrained $(\alpha, \beta, \omega)$. For a negligible flight time $|\alpha|,|\beta|,|\omega| \ll t_{n}^{-1}$ we have

$$
\begin{aligned}
\bar{C}_{3}(\alpha, \beta)= & \sum_{n} R_{n} T_{n}\left(1-2 T_{n} F(\alpha, \beta)\right) 2 e^{4} V / h, \\
F(\alpha, \beta)= & 1-\sum_{\sigma(\alpha \beta \omega)} u(\omega) \hbar / e V \times \\
& {[w(\alpha-e V / \hbar)-w(\beta+e V / \hbar)] / 4, }
\end{aligned}
$$

where we sum over permutations of the constrained set $\alpha \beta \omega$. The temperature-dependence is encoded in a single universal function $F$, which does not depend on the channel transparency. In agreement with previous results 24,25 we find at zero temperature

$$
F(\alpha, \beta)=1-\min \{\max \{|\alpha|,|\beta|,|\alpha+\beta|\},|e V / \hbar|\} \hbar /|e V| .
$$

The zero-frequency limit is of course 35

$$
F(0,0)=1-3 \frac{\sinh U-U}{U(\cosh U-1)}, U=e V / k_{B} T .
$$


The frequency dependence of the function $F$ is plotted in Fig. 3. The form of the plot is motivated by the symmetry of arguments and the constraint $\alpha+\beta+\omega=0$. However, experimentally a verification of the full frequency dependence is a desirable challenge.
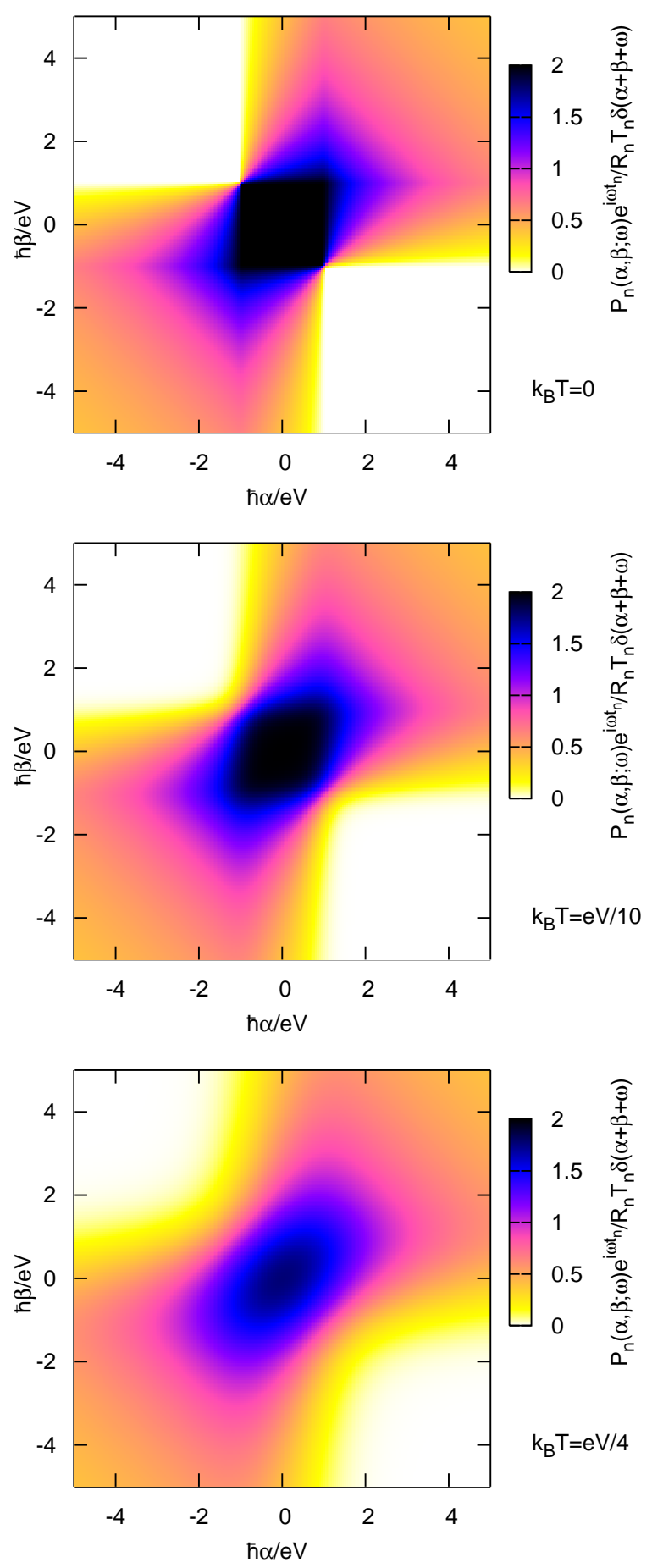

FIG. 2: (Color online) The susceptibility $P_{n}(\alpha, \beta ; \omega)$ at dc voltage defined in (60) for different temperatures. The cross symmetry is preserved for all temperatures.

The effect of flight times on the second cumulant in
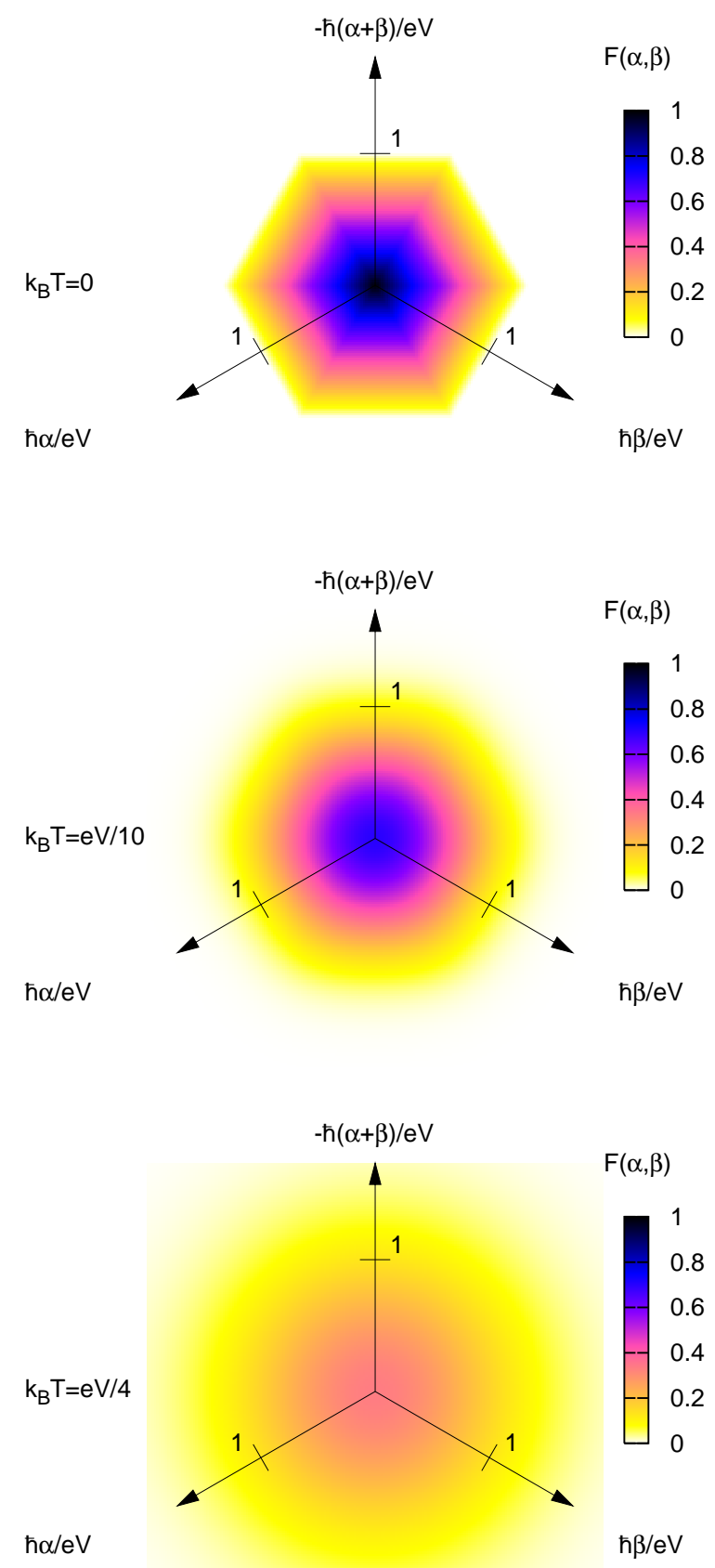

FIG. 3: (Color online) The factor $F(\alpha, \beta)$ defined in (61) for different temperatures. The hexagonal symmetry is preserved for all temperatures.

Eq. (41) was only an additional offset noise, independent of bias voltage. In the case of the third cumulant the terms, which depend on the flight time, depend on voltage, see Eq. (57). For $\omega, \omega^{\prime} \gg t_{n}^{-1}$ we get

$$
\bar{C}_{3}\left(\omega, \omega^{\prime}\right)=F\left(\omega, \omega^{\prime}\right) \bar{C}_{3}(0,0)
$$

so the cumulant drops to zero at large frequencies, contrary to the zero flight time case, where it stays nonzero 
and proportional to $e V$. The recent experiment ${ }^{27}$ does not show any reduction of the third cumulant at high frequency, so the frequency scale defined by the flight time must still be beyond experimental reach. However, there is an environmental correction of a similar magnitude. We will continue the discussion of the third cumulant in Sec. IV.

Lastly, the fourth cumulant can be evaluated in the similar way. Here we present only the result at $\mathrm{eV}=0$,

$$
\begin{aligned}
& \left\langle\left\langle I\left(\omega_{1}\right) I\left(\omega_{2}\right) I\left(\omega_{3}\right) I\left(\omega_{4}\right)\right\rangle\right\rangle= \\
& \sum_{n} \frac{e^{4} R_{n} T_{n}}{8} \delta\left(\sum_{i} \omega_{i}\right) \sum_{\sigma\left(\omega_{i}\right)} e^{2 i t_{n}\left(\omega_{1}+\omega_{2}\right)} u\left(\omega_{3}\right) u\left(\omega_{4}\right) \times \\
& {\left[w\left(\omega_{1}\right)+w\left(\omega_{2}\right)-w\left(\omega_{1}+\omega_{3}\right)-w\left(\omega_{2}+\omega_{3}\right)\right]}
\end{aligned}
$$

where we perform summation over all permutations of the set $\omega_{1}, \ldots, \omega_{4}$. Due to hyperbolic identities, for $t_{n}=$ 0 it reduces to

$$
\sum_{n} \frac{e^{4} R_{n} T_{n}}{2} \delta\left(\sum_{i} \omega_{i}\right) \sum_{i} w\left(\omega_{i}\right)
$$

which agrees with existing results at small reflection or transmission. ${ }^{24}$ One can in principle use this method to find analytic expressions for cumulants of any order, which is beyond the scope of this work.

\section{Tunneling and transparent limit}

In some limits the generating function $\mathcal{S}[\chi]$ can be evaluated exactly. For $\tau \rightarrow 0$, we can make the separation

$$
\mathcal{S}[\chi]=S[\chi, 0]-\int d t \chi^{2}(t) / 2 \tau
$$

where $S[\chi, \phi]$ is defined by (C4). We are particularly interested in the tunneling limit $T_{n} \ll 1$ and the transparent limit $R_{n} \ll 1$ up to terms of the first order in $T_{n}$ $\left(R_{n}\right) \stackrel{24}{2}$ With help of appendix E, we get for $R_{n} \ll 1$

$$
S[\chi, 0]=2 N S_{0}[\chi]+\int \frac{i e V(t)}{\pi \hbar} \sum_{n} \chi\left(t_{+n}\right)+\sum_{n} S_{R n}[\chi]
$$

and for $T_{n} \ll 1$ (tunneling limit),

$$
S[\chi, 0]=\sum_{n} 4 S_{0}\left[\chi_{T n}\right]+\sum_{n} S_{T n}[\chi]
$$

Here, $N$ denotes the number of modes,

$$
S_{0}[\xi]=-\int d t d t^{\prime} \frac{w\left(t-t^{\prime}\right)}{4 \pi} \xi(t) \xi\left(t^{\prime}\right)
$$

is the generating functional of Gaussian vacuum fluctuations and

$$
\begin{aligned}
& S_{A n}[\chi]=-\int \epsilon_{A} \frac{i e V(t)}{\pi \hbar} A_{n} \sin \chi\left(t_{+n}\right) d t+ \\
& \int d t d t^{\prime} \frac{w\left(t-t^{\prime}\right)}{\pi} A_{n}\left\{\epsilon_{A} \chi_{A n}(t) \sin \chi\left(t_{+n}^{\prime}\right)\right. \\
& \left.-2 \sin \frac{\chi\left(t_{+n}\right)}{2} \sin \frac{\chi\left(t_{+n}^{\prime}\right)}{2} e^{i \varphi\left(t^{\prime}, t\right)+D\left(t^{\prime}, t\right)\left[\chi_{A n}\right]}\right\} .
\end{aligned}
$$

The arguments depend on the transmission (reflection) probabilities and are defined by $2 \chi_{A n}(t)=\chi\left(t_{-n}\right)+$ $\epsilon_{A} \chi\left(t_{+n}\right), \epsilon_{R / T}=+/-$. The kernel in the exponent is defined as

$$
\begin{aligned}
& D(a, b)[\xi]=\int_{b}^{a} d s \int d t w(s-t) \xi(t) \\
& =\int\left(e^{i \omega b}-e^{i \omega a}\right) i u(\omega) \xi(-\omega) \frac{d \omega}{2 \pi}=\frac{k_{B} T}{\hbar} \int d t \xi(t) \times \\
& \operatorname{Re}\left[\operatorname{coth}\left(\frac{\pi(a-t)}{\hbar / k_{B} T}+i \epsilon\right)-\operatorname{coth}\left(\frac{\pi(b-t)}{\hbar / k_{B} T}+i \epsilon\right)\right] .
\end{aligned}
$$

One can see that the most significant effect of the flight times in the tunneling limit is Gaussian noise $S_{0}$ that is growing with distance from the contact. This is because $\chi_{T}(t)=\chi\left(t_{-}\right)-\chi\left(t_{+}\right)$vanishes at zero flight time but for large flight times the two parts will become independent and not cancel each other. The noise saturates to the same equilibrium value as in the transparent limit. We stress again, however, that there is no experimental evidence of reaching that timescale so far. The nonGaussian part $S_{A n}$ remains small as it is proportional to $A_{n}$.

In the limit of a vanishing flight time $t_{n}=0$ the above formula for the tunneling case simplifies to

$$
\begin{aligned}
& \frac{S[\chi, 0]}{\sum_{n} T_{n}}=\int i d t \sin \chi(t) e V(t) / \pi \hbar \\
&-\int d t d t^{\prime} \frac{2 w\left(t-t^{\prime}\right)}{\pi} \sin \frac{\chi(t)}{2} \sin \frac{\chi\left(t^{\prime}\right)}{2} \cos \varphi\left(t, t^{\prime}\right)
\end{aligned}
$$

with $w$ and $\varphi$ defined by (23) and (43), respectively.

It is tempting to interpret the last result in terms of a counting statistics. Namely, we might identify terms $e^{ \pm i \chi(t)}$ and $e^{ \pm i \chi(t) / 2 \pm i \chi\left(t^{\prime}\right) / 2}$ with a quasi-charge transfer of $\pm e$ at $t$ and $( \pm e / 2, \pm e / 2)$ at $\left(t, t^{\prime}\right)$, respectively. The fact, that in this expression half-integer charges appear has probably a similar origin as the half-integer charge, which appears in resonant tunneling. ${ }^{36-39}$ Hence, interpreted as a quasi-Poissonian distribution we may identify "probabilities" according to

$$
\begin{aligned}
S \sim & \sum_{\sigma= \pm} p_{\sigma}(t) e^{i \sigma \chi(t)}+ \\
& \sum_{\sigma, \sigma^{\prime}} p_{\sigma \sigma^{\prime}}\left(t, t^{\prime}\right) e^{i \sigma \chi(t) / 2+i \sigma^{\prime} \chi\left(t^{\prime}\right) / 2}
\end{aligned}
$$


By comparing Eq. (73) to Eq. (72) we can read off the transfer "probability" per unit time. These take the form

$$
\begin{aligned}
p_{\sigma}(t) & =\sigma \sum_{n} T_{n} e V(t) d t / 2 \pi \hbar, \\
p_{\sigma \sigma^{\prime}}\left(t, t^{\prime}\right) & =\sigma \sigma^{\prime} \sum_{n} T_{n} d t d t^{\prime} \frac{w\left(t-t^{\prime}\right)}{2 \pi} \cos \varphi\left(t, t^{\prime}\right) .
\end{aligned}
$$

Unfortunately, these rates can be negative, so they cannot be interpreted as a probability. Only a combination of $p_{\sigma}$ and $p_{\sigma \sigma^{\prime}}$ would make sense, which actually happens in usual full counting statistics - valid at long times $\underline{6}$ Hence, the generating function (72) itself does not correspond to a measurable probability. Only after convolution with the Gaussian detection noise we get a real probability.

\section{QUANTUM TAPE}

In section III we have introduced a measurement protocol by means of Kraus operators. Here we go further and want to find a quantum detector that is coupled to a source and registers the time dependence of the source. Our aim is make a quantum tape that translates time dependence into spatial dependence. The tape interacts with the system at a fixed point in space. Then the tape moves far from the point of interaction with the source and afterwards the projection (reading) is applied.

Our quantum tape will be a linear quantum wire or equivalently a massless Josephson transmission line. In this description we have the joint Hamiltonian of the detector and the system

$$
\hat{H}=\hat{H}_{d}+\hat{H}_{I}+\hat{H}_{0} .
$$

Here

$$
\begin{aligned}
& \hat{H}_{d}=\frac{\pi \hbar}{2 e^{2}} \int d s\left(\hat{Q}_{d}^{2}(s)+\hat{I}_{d}^{2}(s)\right) \\
& \hat{H}_{0}=\sum_{\bar{n}} \int d x\left\{V(t) \theta(-x) \hat{Q}_{\bar{n}}(x)\right. \\
& +i \hbar v_{n}\left[\hat{\psi}_{L \bar{n}}^{\dagger}(x) \partial_{x} \hat{\psi}_{L \bar{n}}(x)-\hat{\psi}_{R \bar{n}}^{\dagger}(x) \partial_{x} \hat{\psi}_{R \bar{n}}(x)\right] \\
& \left.+q_{n} \delta(x)\left[\hat{\psi}_{L \bar{n}}^{\dagger}(x) \hat{\psi}_{R \bar{n}}(-x)+\hat{\psi}_{R \bar{n}}^{\dagger}(x) \hat{\psi}_{L \bar{n}}(-x)\right]\right\} \\
& \hat{H}_{I}=-\frac{2 \pi \hbar}{e^{2}} \int d s \lambda(s) \hat{Q}_{d}(s) \hat{Q}
\end{aligned}
$$

with $\lambda(s)=\lambda(-s), \hat{Q}=\sum_{\bar{n}} \int d x f_{n}\left(x / v_{n}\right) \sum_{\bar{n}} \hat{Q}_{\bar{n}}(x)$,

$$
\hat{Q}_{\bar{n}}=\sum_{A=R, L} e \hat{\psi}_{A \bar{n}}^{\dagger}(x) \hat{\psi}_{A \bar{n}}(x) \text {. }
$$

The bosonic operators satisfy (see also the fermionic representation in appendix $\mathrm{F}$ )

$$
\begin{aligned}
& {\left[\hat{I}_{d}(s), \hat{I}_{d}\left(s^{\prime}\right)\right]=\left[\hat{Q}_{d}(s), \hat{Q}_{d}\left(s^{\prime}\right)\right]=0} \\
& {\left[\hat{I}_{d}(s), \hat{Q}_{d}\left(s^{\prime}\right)\right]=-i e^{2} \partial_{s}\left(s-s^{\prime}\right) / \pi .}
\end{aligned}
$$

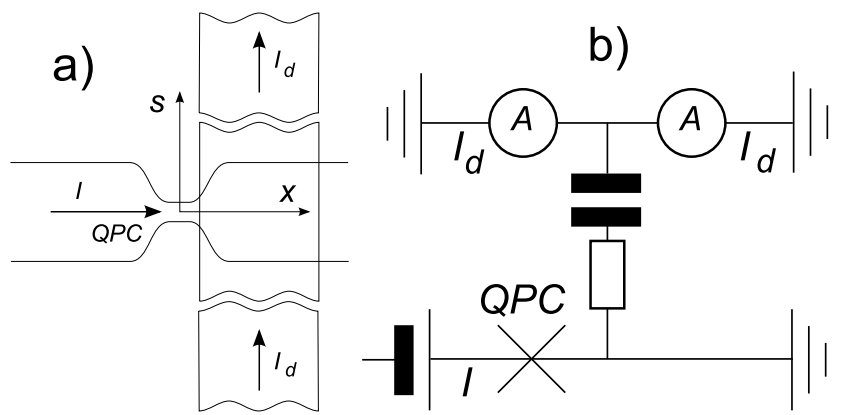

FIG. 4: The system (quantum point contact) coupled to a one-dimensional wire (a) and possible equivalent electric circuit (b). Ammeters, switched on at instant time, measure spatial profile of the current.

We used here time units for the Hilbert space of the detector (Fermi velocity $v_{d}=1$ ). The functions $\lambda$ and $f_{n}$ define the coupling between the detector and the system. Their arguments are in time units and should be nonzero only on one side of the junction. In principle it should be the total charge, i.e. $f_{n}(s)=\theta(s)$, but we have to allow finite range of the coupling. The setup is depicted in Fig. 4.

We also define Heisenberg operators $\hat{A}(t)=$ $\hat{U}^{\dagger}(t) \hat{A} \hat{U}(t)$ with $\hat{U}(t)=\mathcal{T} e^{\int_{0}^{t} \hat{H}\left(t^{\prime}\right) d t^{\prime} / i \hbar}$. The initial density matrix is $\hat{\rho}=\hat{\rho}_{d} \hat{\rho}_{s}$ with $\hat{\rho}_{a} \propto e^{-\hat{H}_{a} / k_{B} T_{a}}$ for $a=d, 0$. The current operator is defined $\hat{I}=\sum_{\bar{n}} e \hat{\psi}_{L \bar{n}}^{\dagger} \hat{\psi}_{L \bar{n}}-L \leftrightarrow$ $R$. We define the auxiliary current operator

$$
\hat{\tilde{I}}(s)=\left(\hat{I}_{d}(s)-\hat{I}_{d}(-s)\right) / 2 .
$$

Now, performing a spatially resolved measurement of $\hat{\tilde{I}}(s, t)$ at $s \gg 0$ (beyond the support of $\lambda(s)$ ) we get the probability density functional

$$
\rho[\tilde{I}]=\operatorname{Tr} \hat{\rho} \delta[\hat{\tilde{I}}-\tilde{I}] .
$$

The generating functional $\mathcal{S}[\chi]=\ln \left\langle e^{\int i \chi(s) \tilde{I}(s-t) d s / e}\right\rangle$ in the limit of weak coupling (neglecting terms $\mathcal{O}\left[\chi \lambda^{2}\right]$ ) will be given by (see appendix F)

$\mathcal{S}[\chi]=\tilde{S}\left[-\dot{\chi} * \lambda, i \chi * \lambda * w_{d} / 2\right]-\int d \omega|\chi(\omega)|^{2} w_{d}(\omega) / 16 \pi^{2}$.

The convolution in the arguments is defined as $(a *$ $\lambda)(\omega)=a(\omega) \lambda(\omega), \dot{\chi}(\omega)=-i \omega \chi(\omega)$. and

$$
\begin{aligned}
& \tilde{S}[\chi, \phi]=\ln \operatorname{Tr} \hat{\rho}_{0} \tilde{\mathcal{T}} e^{\int i d t(\chi(t)+2 \phi(t)) \hat{Q}_{0}(t) / 2 e} \times \\
& \mathcal{T} e^{\int i d t(\chi(t)-2 \phi(t)) \hat{Q}_{0}(t) / 2 e} .
\end{aligned}
$$

Here we used the interaction picture, in which $\hat{A}_{0}(t)=$ $\hat{U}_{0}^{\dagger}(t) \hat{A} \hat{U}_{0}(t)$ with $\hat{U}_{0}(t)=\mathcal{T} e^{\int_{0}^{t} \hat{H}_{0}\left(t^{\prime}\right) d t^{\prime} / i \hbar}$. We get the expected detection picture - the measured signal $\tilde{I}(\omega)$ is proportional to $\lambda(\omega) I_{0}(\omega)$ with $\hat{I}_{0}(t)=d \hat{Q}(t) / d t=$ $i\left[\hat{H}_{0}(t), \hat{Q}_{0}(t)\right] / \hbar$. The contribution from $I_{0}$ is much 
weaker $(\lambda \ll 1)$ than the internal Gaussian noise of the detector - the last term in (81). To evaluate (82), we can apply the FCS formalism developed in Sec. III and App. A for $\hat{H}_{I}=\int d x \sum_{\bar{n}} \hat{Q}_{\bar{n}}(x) V_{s \bar{n}}(x, t)$ with the bias $V_{s \bar{n}}(x, t)+V(t) \theta(-x)$,

$$
V_{s \bar{n}}(x, t)=\hbar \phi(t) f_{n}\left(x / v_{n}\right) / e .
$$

The averages measured at the detector are a combination of its own noise, the noise of the system and the response of the system due to the detector. The average

$$
\langle\tilde{I}(\omega)\rangle=\sum_{n} \tilde{g}_{n}(\omega) T_{n} e^{2} V(\omega) / \pi \hbar
$$

with $\tilde{g}_{n}=g_{n}\left(1+\mathcal{O}\left[g^{2}\right]\right), g_{n}(\omega)=-i \omega \lambda(\omega) f_{n}(\omega)$. The observed fluctuation spectral density of $\tilde{I}$ is $C_{2}\left(\omega, \omega^{\prime}\right)=\left\langle\delta \tilde{I}(\omega) \delta \tilde{I}\left(\omega^{\prime}\right)\right\rangle=2 \pi e^{2} \delta\left(\omega+\omega^{\prime}\right) P(\omega)+$ $\sum_{n} e^{2} \tilde{g}_{n}(\omega) \tilde{g}_{n}\left(\omega^{\prime}\right) P_{n}\left(\omega, \omega^{\prime}\right)$. It contains a voltage independent contribution

$$
\begin{aligned}
& P(\omega)=w_{d}(\omega) / 4 \pi-\sum_{n} R_{n} T_{n} w(\omega)\left|g_{n}(\omega)\right|^{2} / \pi \\
& +\sum_{n}\left[w(\omega)-w_{d}(\omega)\right]\left[\left|g_{n}(\omega)\right|^{2}-R_{n} \operatorname{Re} g_{n}^{2}(\omega)\right] / \pi,
\end{aligned}
$$

where $w(\omega)=\omega \operatorname{coth}\left(\hbar \omega / 2 k_{B} T_{0}\right)$. The second part is

$$
\begin{aligned}
& P_{n}\left(t, t^{\prime}\right)=R_{n} T_{n} \frac{w\left(t-t^{\prime}\right)}{\pi \Gamma_{n}\left(t-t^{\prime}\right)} \cos \int_{t}^{t^{\prime}} d u \frac{e V(u)}{\hbar}, \\
& \ln \Gamma_{n}(t)=\int d \omega \frac{2 \sin ^{2}(\omega t)}{(2 \pi \omega)^{2}}\left[w_{d}(\omega)\left|g_{n}(\omega)\right|^{2}+\mathcal{O}\left[g^{4}\right]\right] .
\end{aligned}
$$

The excess noise - i.e. the voltage-dependent part of $C_{2}$ - is contained in (86). Note that this result is valid for an arbitrary time-dependent bias voltage. In the limit of weak coupling, viz. $g_{n} \ll 1$, we can take $\Gamma(t) \simeq 1$ and the excess noise agrees with the symmetrized quantum noise $\stackrel{4,34}{=}$ It is a tiny contribution on top of a huge background noise $P(\omega)$. The background noise is also seen in experiments but it cannot be distinguished from the amplifier's noise. Measurements of the voltage de-

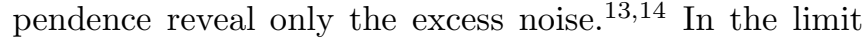
of strong coupling, $g_{n} \gg 1$, the noise is heavily affected by the detector's backaction. The excess noise is affected through $\Gamma(t)>1$ and gets a significant correction, similar to that in Sec. III, which vanishes for high voltage since $\Gamma(t \rightarrow 0)=1$.

The situation is different for the third cumulant. In the limit of weak coupling we keep only terms to the lowest order in $g_{n}$, which gives

$$
\begin{aligned}
& C_{3}\left(\omega_{1}, \omega_{2}, \omega_{3}\right)=\left\langle\delta \tilde{I}\left(\omega_{1}\right) \delta \tilde{I}\left(\omega_{2}\right) \delta \tilde{I}\left(\omega_{3}\right)\right\rangle= \\
& \sum_{n} e^{3} \prod_{l} g_{n}\left(\omega_{l}\right)\left\{R_{n} T_{n}\left(R_{n}-T_{n}\right) \frac{e V\left(\omega_{1}+\omega_{2}+\omega_{3}\right)}{\pi \hbar}\right. \\
& \left.+\sum_{\sigma(i j k)}\left(2 T_{n}+q_{n}\left(\omega_{k}\right)-1\right) P_{n}\left(\omega_{i}, \omega_{j} ; \omega_{k}\right) w\left(\omega_{k}\right) / 4\right\}
\end{aligned}
$$

FIG. 5: The high-frequency third cumulant of current fluctuations in the tunneling limit at zero temperature for different values of $q=q_{n}$. The average current is $I_{0}=2 e^{2} \sum_{n} T_{n} V / h$, we took $|\Omega| \ll(\omega, e V / \hbar)$ and assumed a mode-independent coupling $g=g_{n}$. Note that $\operatorname{Re} q \leq 0$ at $\vartheta=0$ and $q=0$ at $k_{B} T_{d}=0$.

for the susceptibility $P_{n}(\alpha, \beta ; s)$ defined by (58), $q_{n}(\omega)=$ $e^{i \vartheta_{n}(\omega)}\left[1-w_{d}(\omega) / w(\omega)\right]$ and $\vartheta_{n}(\omega)=-2 \arg g_{n}(\omega)$. The inner summation runs over all permutations of the set 123. Note that $q_{n}$ leads to corrections that are indistinguishable from the influence of an environment described by (59) (up to factors $g_{n}$ ). The detection dependent part $g_{n}(\omega)\left(q_{n}(\omega)-1\right) / \hbar$ from (87) can be effectively absorbed into $b_{0}$ in (59). Hence, the detector can be interpreted as an example of some environment.

Another interesting observation is that all cumulants, except the first term in (85), vanish at equilibrium between detector and the system, namely for $\mathrm{eV}=0$ and $k_{B} T_{0}=k_{B} T_{d}$. On the other hand it is obvious because no information transfer is possible at equilibrium as the entropy is already maximized. An efficient detector cannot be in equilibrium with the measured system.

Let us finally consider two limiting cases. Analogously to (61) and (62), for $k_{B} T_{0}=0$ and $\Omega \ll(\omega,|e V| / \hbar)$ we get from (87)

$$
\begin{aligned}
& \bar{C}_{3}(\Omega, \omega)=\sum_{n} g_{n}(\Omega)\left|g_{n}(\omega)\right|^{2} 2 R_{n} T_{n} \frac{e^{4} V}{h} \times \\
& {\left[1-2 T_{n}+\left(2 T_{n}-1+\operatorname{Re} q_{n}(\omega)\right) \min \left(1, \frac{\hbar|\omega|}{|e V|}\right)\right] .}
\end{aligned}
$$

This result is plotted for different $q_{n}$ in Fig. 5 in the tunneling limit, $T_{n} \ll 1$. We see, that $q_{n}$ induces a step in $\bar{C}_{3}$ for $e V=\hbar \omega$. For arbitrary $k_{B} T_{0}$ and $\left|\omega_{k}\right| \ll|e V| / \hbar$, we have

$$
\begin{aligned}
& \bar{C}_{3}\left(\omega_{1}, \omega_{2}\right)=\frac{e^{4} V}{h} \sum_{n} 2 R_{n} T_{n} \prod_{k} g_{n}\left(\omega_{k}\right) \times \\
& {\left[1-2 T_{n}+\left(2 T_{n}-1+\sum_{k} q_{n}\left(\omega_{k}\right) / 3\right)(1-F(0,0))\right]}
\end{aligned}
$$

with $F(0,0)$ given by (63) in agreement with the environmental correction $\stackrel{10}{\underline{ }}$ We see, that the behavior of the 
third cumulant is different from the noise. Even in the weak coupling limit it may get a significant quantum correction due to the detector.

\section{A. Many tapes}

We have seen in Eq.81) that the outcome of the measurement is always correlated with fluctuations in the tape as the second argument of $\tilde{S}$ is non-zero. This can be avoided by means of many tapes, namely

$$
\hat{H}=\hat{H}_{0}+\frac{\pi \hbar}{2 e^{2}} \sum_{c=1}^{N} \int\left[\hat{Q}_{c}^{2}(s)+\hat{I}_{c}^{2}(s)-4 \lambda_{c}(s) \hat{Q}_{c}(s) \hat{Q}\right] d s .
$$

with

$$
\begin{aligned}
& {\left[\hat{I}_{a}(s), \hat{Q}_{b}\left(s^{\prime}\right)\right]=-i e^{2} \delta_{a b} \partial_{s} \delta\left(s-s^{\prime}\right) / \pi,} \\
& {\left[\hat{I}_{a}(s), \hat{I}_{b}\left(s^{\prime}\right)\right]=\left[\hat{Q}_{a}(s), \hat{Q}_{b}\left(s^{\prime}\right)\right]=0 .}
\end{aligned}
$$

We also redefine

$$
\hat{\tilde{I}}(s)=\sum_{c}\left(\hat{I}_{c}(s)-\hat{I}_{c}(-s)\right) .
$$

The resulting generating function (F11) has the new form in the limit of weak coupling,

$$
\begin{aligned}
& \mathcal{S}[\chi]=\tilde{S}\left[-\sum_{c} \dot{\chi} * \lambda_{c}, \sum_{c} i \chi * \lambda_{c} * w_{c} / 2\right] \\
& -\int d \omega|\chi(\omega) / 4 \pi|^{2} \sum_{c} w_{c}(\omega) .
\end{aligned}
$$

The results for the cumulants differ from the single tape case as follows. In the definition of $g_{n}$ below (84) we have to replace $\lambda \rightarrow \sum_{c} \lambda_{c}$. In (85) we change the first term $w_{d} \rightarrow \sum_{c} w_{c}$. We also change $w_{d} \rightarrow \sum_{c} \lambda_{c} w_{c} / \sum_{c} \lambda_{c}$ in the second line of (85) and the definition of $q_{n}$ below (87). This change makes in principle arbitrarily large $q_{n}$ (positive or negative) possible even at $\vartheta_{n}=0$. On the other hand a simple model of environment ${ }^{10}$ can give effectively $q_{n} \leq 1$ when comparing (87) and (59), while the case $q_{n}>1$ has been observed in the recent experiment. ${ }^{27}$ The latter suggests that the actual detection scheme or model of environment is more complicated.

Let us consider a very special case of tapes at high temperatures $1 / \lambda_{c} \tau \gg k_{B} T_{c} / \hbar \gg 1 / \tau$, where $\tau$ is a certain time resolution so that the measurable range of frequencies is $|\tau \omega| \ll 1$. If $\sum_{c} \lambda_{c} k_{B} T_{c}=0$ (but $\sum_{c} \lambda_{c} \neq 0$ ) then we can write

$$
\mathcal{S}[\chi]=\tilde{S}\left[-\sum_{c} \dot{\chi} * \lambda_{c}, 0\right]-\int d \omega|\chi(\omega)|^{2} \sum_{c} w_{c}(\omega) / 16 \pi^{2},
$$

so that the system-detector correlation cancels (0 in the second argument of $\tilde{S})$. The detector model becomes then classical while still gaining quantum information. Such a situation is close to the POVM model from Sec. III in the limit of weak coupling because the detector's noise (last term in (94)) becomes white and decouples from the system's signal.

\section{CONCLUSIONS}

We have presented detection models of time-resolved quantum detection of current in mesoscopic junction. One can make the measurement by means of either a partial projection (Kraus operator - POVM) or a full projection on a weakly coupled transmission line - quantum tape. It is difficult to separate the backaction of the detector from the signal of the system. Nevertheless, the generality of the presented method makes it applicable to a wide range of types of measurement.

Applied to a quantum point contact, both models give the expected expressions for current and noise if the background noise is subtracted. The voltage-dependent part of the noise is independent of the detector in the weak coupling limit. On the other hand, the third cumulant may contain significant voltage-dependent corrections even in the weak coupling limit. The correction is indistinguishable from the effect of the environment. Hence, the high frequency measurement in mesoscopic junctions always contains a detection-dependent contribution. Experimentally, an independent determination of the coupling parameters would help to distinguish between the effects of the detection process and the environmental backaction.

We have also derived an analytical tool to calculate frequency-dependent cumulants for a mesoscopic junction with energy-independent transmission. An important result is to identify the noise susceptibility in the expression for the third cumulant at high frequency. The method works well both in time and frequency domain and can be extended to other complicated correlations.

\section{Acknowledgments}

We are grateful to J. Gabelli and B. Reulet for discussions. We acknowledge financial support from the German Research Foundation (DFG) through SFB 767 and SP 1285 Semiconductor Spintronics.

\section{Appendix A}

In our model of the quantum point contact, we make the approximation of a linear spectrum and a short range scattering potential. Both assumptions lead to singular behaviour and we have to regularize some terms in the Hamiltonian and describe the details here.

The short range potential scattering is introduced in Eq. (8) and we would like to relate this term to the 
scattering matrix. One of the possible regularizations of (8) is

$$
\sum_{\bar{n}} \int d x z_{n}^{\prime}(x) v_{n}\left[\hat{\psi}_{L \bar{n}}^{\dagger}(x) \hat{\psi}_{R \bar{n}}(x)+\hat{\psi}_{R \bar{n}}^{\dagger}(x) \hat{\psi}_{L \bar{n}}(x)\right]
$$

with the function $z_{n}(x) \rightarrow q_{n} \theta(x)$. For $\left|k_{n} x\right| \ll 1$, the wave eigenfunctions satisfy

$$
\begin{aligned}
& i \hbar \partial_{x} \psi_{L \bar{n}}(x)+z_{n}^{\prime}(x) \psi_{R \bar{n}}(x)=0, \\
& -i \hbar \partial_{x} \psi_{R \bar{n}}(x)+z_{n}^{\prime}(x) \psi_{L \bar{n}}(x)=0 .
\end{aligned}
$$

We can change variables $x \rightarrow z(x)$,

$$
\begin{aligned}
& i \hbar \partial_{z_{n}} \psi_{L \bar{n}}+\psi_{R \bar{n}}=0, \\
& -i \hbar \partial_{z_{n}} \psi_{R \bar{n}}+\psi_{L \bar{n}}=0,
\end{aligned}
$$

which gives the solution

$$
\begin{aligned}
& \psi_{L \bar{n}}=c_{+} e^{z_{n} / \hbar}+c_{-} e^{-z_{n} / \hbar} \\
& \psi_{R \bar{n}}=-i c_{+} e^{z_{n} / \hbar}+i c_{-} e^{-z_{n} / \hbar} .
\end{aligned}
$$

By taking $\psi_{L \bar{n}}\left(z_{n}=q_{n}\right)=0, \psi_{R \bar{n}}\left(z_{n}=0\right)=1$, we get

$$
\begin{aligned}
& \tilde{t}_{n}=\psi_{R \bar{n}}\left(z_{n}=q_{n}\right)=\frac{1}{\cosh \left(q_{n} / \hbar\right)}, \\
& \tilde{r}_{n}=\psi_{L \bar{n}}\left(z_{n}=0\right)=-i \tanh \left(q_{n} / \hbar\right) .
\end{aligned}
$$

The phase factor in $\tilde{t}_{n}$ is completely irrelevant in our approximation. However, it can be added by a constant, symmetric bias potential.

For the future convenience we shall use a little different regularization of the second term in (8), namely

$$
\sum_{\bar{n}} \int d x z_{n}^{\prime}\left(x / v_{n}\right)\left[\hat{\psi}_{L \bar{n}}^{\dagger}(x) \hat{\psi}_{R \bar{n}}(-x)+\hat{\psi}_{R \bar{n}}^{\dagger}(-x) \hat{\psi}_{L \bar{n}}(x)\right] .
$$

It gives $\tilde{t}_{n}=\cos \left(q_{n} / \hbar\right)$ and $\tilde{r}_{n}=i \sin \left(q_{n} / \hbar\right)$. The regularized Hamiltonian (12) takes the form

$$
\begin{aligned}
& \hat{H}=\hat{H}_{0}+\sum_{\bar{n}} \int d s 2 z_{n}^{\prime}(s) \hat{I}_{3 \bar{n}}(s) / e \\
& +\sum_{\bar{n}, \pm} \int d s V\left( \pm s v_{n}, t\right)\left[\hat{I}_{0 \bar{n}}(s) \pm \hat{I}_{1 \bar{n}}(s)\right]
\end{aligned}
$$

To derive (14) and (15), we recall the standard bosonization scheme for a $1 \mathrm{D}$ system of noninteracting fermions 30 Let us define

$$
\hat{\psi}_{L \bar{n}}(k)=\int \frac{d x}{\sqrt{2 \pi}} e^{i k x} \hat{\psi}_{L \bar{n}}(x)
$$

We assume that the excitations of the Fermi sea are finite in the sense that levels deep below and high above the Fermi level are always occupied and empty, respectively. This leads to

$$
\hat{\psi}_{L \bar{n}}(k \rightarrow-\infty), \hat{\psi}_{L \bar{n}}^{\dagger}(k \rightarrow \infty) \rightarrow 0
$$

when acting on the density matrix standing to the right of these operators. Next we construct the operators

$$
\begin{aligned}
& \hat{A}(x)=\hat{\psi}_{L \bar{n}}^{\dagger}(x) \hat{\psi}_{L \bar{n}}(x)=\int \frac{d k}{\sqrt{2 \pi}} e^{-i k x} \hat{A}(k), \\
& \hat{A}(k)=\int \frac{d q}{\sqrt{2 \pi}} \hat{\psi}_{L \bar{n}}^{\dagger}(q) \hat{\psi}_{L \bar{n}}(q+k) .
\end{aligned}
$$

The last operator needs to be regularized as

$$
\hat{A}(k)=\int \frac{d q}{\sqrt{2 \pi}} g(q) \hat{\psi}_{L \bar{n}}^{\dagger}(q) \hat{\psi}_{L \bar{n}}(q+k),
$$

where $g(q)=1$ for $|q| \ll \Lambda$ and $g(q) \rightarrow 0$ for $q \gg \Lambda$, with $\Lambda$ denoting some cutoff larger than all relevant wave scales. Then

$$
\left\{\hat{\psi}_{L \bar{n}}^{\dagger}(q), \hat{\psi}_{L \bar{n}}(k)\right\}=\delta(q-k) .
$$

Hence,

$$
\begin{aligned}
& {\left[\hat{A}(k), \hat{A}\left(k^{\prime}\right)\right]=\int \frac{d q}{2 \pi} \hat{\psi}_{L \bar{n}}^{\dagger}\left(q+k+k^{\prime}\right) \hat{\psi}_{L \bar{n}}(q) \times} \\
& g(q)\left(g\left(q+k^{\prime}\right)-g(q+k)\right) .
\end{aligned}
$$

For $|q|,|k|,\left|k^{\prime}\right| \ll \Lambda$ the above expression gives zero because $g\left(q+k^{\prime}\right)=g(q+k)$. Also for $|q| \gg \Lambda$, it zero due to $q(q)=0$. For $q \sim-\Lambda$ we get again zero due to (A9). The only nonzero terms come from $q \sim \Lambda$. We can use here also (A9) but only after changing the order of the operators $\hat{\psi}$ and $\hat{\psi}^{\dagger}$. The net contribution is the anticommutator for $q>0$. Hence,

$$
\begin{aligned}
& {\left[\hat{A}(k), \hat{A}\left(k^{\prime}\right)\right]=} \\
& \int_{0}^{\infty} \frac{d q}{2 \pi} \delta\left(k+k^{\prime}\right) g(q)\left(g\left(q+k^{\prime}\right)-g(q+k)\right)= \\
& =\delta\left(k+k^{\prime}\right) \int_{0}^{\infty} \frac{d q}{2 \pi} g(q)(g(q-k)-g(q+k))= \\
& \delta\left(k+k^{\prime}\right) \int_{0}^{k} \frac{d q}{2 \pi} g(q) g(q-k)=k \delta\left(k+k^{\prime}\right) / 2 \pi .
\end{aligned}
$$

In the last line we used the fact that $g(q)=1$ for $q, k \ll$ $\Lambda$. By taking the Fourier transform we get

$$
\left[\hat{A}(x), \hat{A}\left(x^{\prime}\right)\right]=i \partial_{x} \delta\left(x-x^{\prime}\right) / 2 \pi .
$$

\section{Appendix B}

The current operators defined in (11) can be found exactly by a solution of their respective equations of motion. We have the following Heisenberg equations (24) for (11),

$$
\begin{aligned}
& D \hat{I}_{0 \bar{n}}(s, t)=e^{2} \partial_{s} V_{+}\left(s v_{n}, t\right) / 4 \pi, \\
& D \hat{I}_{1 \bar{n}}(s, t)=-2 z_{n}^{\prime}(s) \hat{I}_{2 \bar{n}}(s, t)+e^{2} \partial_{s} V_{-}\left(s v_{n}, t\right) / 4 \pi, \\
& D \hat{I}_{2 \bar{n}}(s, t)=2 z_{n}^{\prime}(s) \hat{I}_{1 \bar{n}}(s, t)-e V_{-}\left(s v_{n}, t\right) \hat{I}_{3 \bar{n}}(s, t), \\
& D \hat{I}_{3 \bar{n}}(s, t)=e V_{-}\left(s v_{n}, t\right) \hat{I}_{2 \bar{n}}(s, t),
\end{aligned}
$$


where $D=\hbar\left(\partial_{t}-\partial_{s}\right)$ and $V_{ \pm}(x, t)=V(x, t) \pm V(-x, t)$. For $s \rightarrow+\infty$ we have asymptotic equilibrium operators

$$
\hat{I}_{j \bar{n}}(s, t) \rightarrow \hat{I}_{j \bar{n}}\left(t_{s}\right)
$$

for $j=0,1,2,3$ and $t_{s}=t+s$. The operator $\hat{I}_{0}$ decouples from the other currents and has the general solution

$$
\hat{I}_{0 \bar{n}}(s, t)=\hat{I}_{0 \bar{n}}\left(t_{s}\right)+\frac{e^{2}}{4 \pi \hbar} \partial_{s} \int_{s}^{\infty} d s^{\prime} V_{+}\left(s^{\prime} v_{n}, t+s-s^{\prime}\right) .
$$

For $s>0$ we have

$$
\begin{aligned}
& \hat{I}_{1 \bar{n}}(s, t)=\hat{I}_{1 \bar{n}}\left(t_{s}\right)+\frac{e}{4 \pi} \partial_{s} \Phi_{n}(s, t), \\
& \hat{I}_{2 \bar{n}}(s, t)=\hat{I}_{2 \bar{n}}\left(t_{s}\right) \cos \Phi_{n}(s, t)-\hat{I}_{3 \bar{n}}\left(t_{s}\right) \sin \Phi_{n}(s, t), \\
& \hat{I}_{3 \bar{n}}(s, t)=\hat{I}_{3 \bar{n}}\left(t_{s}\right) \cos \Phi_{n}(s, t)+\hat{I}_{2 \bar{n}}\left(t_{s}\right) \sin \Phi_{n}(s, t), \\
& \Phi_{n}(s, t)=\frac{e}{\hbar} \int_{s}^{\infty} d s^{\prime} V_{-}\left(s^{\prime} v_{n}, t+s-s^{\prime}\right)
\end{aligned}
$$

and for $s<0$

$$
\begin{aligned}
& \hat{I}_{1 \bar{n}}(s, t)=\hat{I}_{1 \bar{n}}^{0}\left(t_{s}\right)+\frac{e}{4 \pi} \partial_{s} \Phi_{+n}(s, t), \\
& \hat{I}_{2 \bar{n}}(s, t)=\hat{I}_{2 \bar{n}}^{0}\left(t_{s}\right) \cos \Phi_{+n}(s, t)-\hat{I}_{3 \bar{n}}^{0}\left(t_{s}\right) \sin \Phi_{+n}(s, t), \\
& \hat{I}_{3 \bar{n}}(s, t)=\hat{I}_{3 \bar{n}}^{0}\left(t_{s}\right) \cos \Phi_{+n}(s, t)+\hat{I}_{2 \bar{n}}^{0}\left(t_{s}\right) \sin \Phi_{+n}(s, t), \\
& \Phi_{+n}(s, t)=\Phi_{n}(s, t)-\Phi_{n}\left(t_{s}\right), \Phi_{n}(t)=\Phi_{n}(0, t), \quad(\mathrm{B} 5)
\end{aligned}
$$

with the boundary conditions

$$
\begin{aligned}
& \hat{I}_{1 \bar{n}}^{0}(s)=\left(T_{n}-R_{n}\right) \hat{I}_{1 \bar{n}}\left(0_{+}, s\right)-2 \sqrt{R_{n} T_{n}} \hat{I}_{2 \bar{n}}\left(0_{+}, s\right), \\
& \hat{I}_{2 \bar{n}}^{0}(s)=\left(T_{n}-R_{n}\right) \hat{I}_{2 \bar{n}}\left(0_{+}, s\right)+2 \sqrt{R_{n} T_{n}} \hat{I}_{1 \bar{n}}\left(0_{+}, s\right), \\
& \hat{I}_{3 \bar{n}}^{0}(s)=\hat{I}_{3}\left(0_{+}, s\right) .
\end{aligned}
$$

In particular for $s<0$

$$
\begin{aligned}
& \hat{I}_{1 \bar{n}}(s, t)=\left(T_{n}-R_{n}\right) \hat{I}_{1 \bar{n}}\left(t_{s}\right) \\
& +\frac{e}{4 \pi} \partial_{s}\left(\Phi_{n}(s, t)-2 R_{n} \Phi_{n}\left(t_{s}\right)\right)-2 \sqrt{R_{n} T_{n}} \hat{J}_{\bar{n}}^{\Phi}\left(t_{s}\right)
\end{aligned}
$$

with

$$
\hat{J}_{\bar{n}}^{\Phi}(s)=\hat{I}_{2 \bar{n}}(s) \cos \Phi_{n}(s)-\hat{I}_{3 \bar{n}}(s) \sin \Phi_{n}(s) .
$$

The great advantage of the above equations is that the Heisenberg current operators can be represented by combinations of equilibrium operators. The same applies to averages and facilitates the calculation of higher cumulants.

\section{Appendix C}

To evaluate the noise correction due to the detetctor in the POVM model we employ the the formalism developed previously. We have to perform a number of path integrals to find the final analytical expressions for the noise. We start by splitting Eq. (29) into two parts

$$
\begin{aligned}
& \hat{I}_{R}(t)=\sum_{\bar{n}}\left[\hat{I}_{R \bar{n}}^{\mathrm{in}}(t)+\hat{I}_{R \bar{n}}^{\mathrm{out}}(t)\right], \quad(\mathrm{C} 1) \\
& \hat{I}_{R \bar{n}}^{\text {out }}(t)=\int d x g\left(x+x_{R}\right)\left[\hat{I}_{0 \bar{n}}\left(x_{n}, t\right)-\hat{I}_{1 \bar{n}}\left(x_{n}, t\right)\right], \\
& \hat{I}_{R \bar{n}}^{\mathrm{in}}(t)=\int d x g\left(x-x_{R}\right)\left[-\hat{I}_{0 \bar{n}}\left(x_{n}, t\right)-\hat{I}_{1 \bar{n}}\left(x_{n}, t\right)\right],
\end{aligned}
$$

with $x_{n}=x / v_{n}$. To simplify (34), we will use the commutators

$$
\begin{aligned}
& {\left[\hat{I}_{R \bar{n}}^{\mathrm{in}}(t), \hat{I}_{R \bar{n}}^{\mathrm{out}}\left(t^{\prime}\right)\right]=0 \text { for } t>t^{\prime}} \\
& {\left[\hat{I}_{R \bar{n}}^{\mathrm{in}}(t), \hat{I}_{R \bar{n}}^{\mathrm{in}}\left(t^{\prime}\right)\right]=\left[\hat{I}_{R \bar{n}}^{\mathrm{out}}(t), \hat{I}_{R \bar{n}}^{\mathrm{out}}\left(t^{\prime}\right)\right]=\frac{i e^{2}}{2 \pi} h_{n}^{\prime}\left(t-t^{\prime}\right) .}
\end{aligned}
$$

with $h_{n}$ defined by (35). Using the Baker-Hausdorff formula $e^{\hat{A}} e^{\hat{B}}=e^{\hat{A}+\hat{B}} e^{[\hat{A}, \hat{B}] / 2}$ we find

$$
\begin{aligned}
& \mathcal{T} e^{\int \frac{i d t}{2 e} \xi(t) \hat{I}_{R}(t)}=\prod_{\bar{n}} \exp \int \frac{i d t}{2 e} \xi(t) \hat{I}_{R \bar{n}}^{\text {out }}(t) \times \quad(\mathrm{C} 3) \\
& \exp \int \frac{i d t}{2 e} \xi(t) \hat{I}_{R \bar{n}}^{\mathrm{in}}(t) \exp \int \frac{-i d t d t^{\prime}}{8 \pi} \xi(t) \xi\left(t^{\prime}\right) \theta_{n}\left(t-t^{\prime}\right) .
\end{aligned}
$$

Using similar transformations we get

$$
e^{S[\chi, \phi]}=\operatorname{Tr} \hat{\rho} \prod_{\bar{n}} \hat{U}_{\bar{n}}^{\mathrm{in}} \hat{U}_{\bar{n}}^{\text {out }} \hat{U}_{\bar{n}}^{\mathrm{in}} e^{i \vartheta_{n}}
$$

where

$$
\begin{aligned}
& \hat{U}_{\bar{n}}^{\mathrm{in}}=\exp \int \frac{i d t}{2 e} \chi(t) \hat{I}_{R \bar{n}}^{\mathrm{in}}(t), \\
& \hat{U}_{\bar{n}}^{\text {out }}=\exp \int \frac{i d t}{e} \chi(t) \hat{I}_{R \bar{n}}^{\phi}(t), \\
& \hat{I}_{R \bar{n}}^{\phi}(t)=\hat{U}_{\phi \bar{n}} \hat{I}_{R \bar{n}}^{\text {out }}(t) \hat{U}_{\phi \bar{n}}^{\dagger}, \\
& \hat{U}_{\phi \bar{n}}=\exp \int \frac{i d t}{e} \phi(t) \hat{I}_{R \bar{n}}^{\mathrm{in}}(t), \\
& \vartheta_{n}=\int \frac{d t d t^{\prime}}{\pi} \chi(t) \phi\left(t^{\prime}\right) \theta_{n}\left(t-t^{\prime}\right)
\end{aligned}
$$

with $\theta_{n}$ defined by (35). Next, to find the noise (39), we need the help of the relation

$$
\int D \phi \exp \left(-\int d t\left[\frac{2 \phi^{2}(t)}{\tau}+i \phi(t) \xi(t)\right]\right)=e^{-\int \frac{\tau d t}{8} \xi^{2}(t)},
$$

which gives

$$
\begin{aligned}
& P(a, b)=\delta(a-b) / \tau+ \\
& \int d t \sum_{n m} \frac{\tau}{\pi^{2}} \theta_{n}(a-t) \theta_{m}(b-t)+\int D \phi e^{-\int \frac{2 d t}{\tau} \phi^{2}(t)} \\
& \left\{\left[\frac{1}{2} \sum_{\bar{n}} \operatorname{Tr} \hat{\rho}\left(\delta \hat{I}_{R \bar{n}}^{\mathrm{in}}(a)+\delta \hat{I}_{R \bar{n}}^{\phi}(a)\right)\left(\delta \hat{I}_{R \bar{n}}^{\mathrm{in}}(b)+\delta \hat{I}_{R \bar{n}}^{\phi}(b)\right)\right.\right. \\
& \left.\left.+\sum_{n \bar{m}} \int \frac{2 d t}{\pi} \phi(t) \theta_{n}(a-t) \operatorname{Tr} \hat{\rho} \hat{I}_{R \bar{m}}^{\phi}(b)\right]+a \leftrightarrow b\right\}
\end{aligned}
$$


and $\delta \hat{A}=\hat{A}-\operatorname{Tr} \hat{\rho} \hat{A}$. Finally, we get

$$
\begin{aligned}
& \delta \hat{I}_{R \bar{n}}^{\mathrm{in}}(t)=-\hat{I}_{0 \bar{n}}\left(t_{+n}\right)-\hat{I}_{1 \bar{n}}\left(t_{+n}\right), \\
& \delta \hat{I}_{R \bar{n}}^{\phi}(t)=-\frac{e R_{n}}{2 \pi} \phi^{\prime}\left(t-2 t_{n}\right)+ \\
& \hat{I}_{0 \bar{n}}\left(t_{-n}\right)-\left(T_{n}-R_{n}\right) \hat{I}_{1 \bar{n}}\left(t_{-n}\right)+2 \sqrt{R_{n} T_{n}} \hat{J}_{\bar{n}}^{\phi}\left(t_{-n}\right), \\
& \phi_{n}(t)=\Phi(t)+\int \sqrt{2} d t^{\prime} \phi\left(t_{-n}+t^{\prime}\right) h_{n}\left(\sqrt{2} t^{\prime}\right)
\end{aligned}
$$

where $\hat{J}$ is defined by (B7) with $\Phi_{n}$ replaced by $\phi_{n}$.

The case of many detectors (Eqs. (49)-(55)) is analogous,

$$
\begin{aligned}
& \hat{I}_{A}(t)=\sum_{\bar{n}}\left[\hat{I}_{A \bar{n}}^{\text {in }}(t)+\hat{I}_{A \bar{n}}^{\text {out }}(t)\right], \\
& \hat{I}_{A \bar{n}}^{\text {out }}(t)=\int d x g\left(x+\left|x_{A}\right|\right)\left[\epsilon_{A} \hat{I}_{0 \bar{n}}\left(x_{n}, t\right)-\hat{I}_{1 \bar{n}}\left(x_{n}, t\right)\right], \\
& \hat{I}_{A \bar{n}}^{\text {in }}(t)=\int d x g\left(x-\left|x_{A}\right|\right)\left[-\epsilon_{A} \hat{I}_{0 \bar{n}}\left(x_{n}, t\right)-\hat{I}_{1 \bar{n}}\left(x_{n}, t\right)\right]
\end{aligned}
$$

with $\epsilon_{A}=\operatorname{sgn} x_{A}$. In Eq. (C44) we put

$$
\begin{aligned}
& \hat{U}_{\bar{n}}^{\mathrm{in}}=\exp \int \frac{i d t}{2 e} \sum_{A} \chi_{A}(t) \hat{I}_{A \bar{n}}^{\mathrm{in}}(t), \\
& \hat{U}_{\bar{n}}^{\mathrm{out}}=\exp \int \frac{i d t}{e} \sum_{A} \chi_{A}(t) \hat{I}_{A \bar{n}}^{\phi}(t), \\
& \hat{I}_{A \bar{n}}^{\phi}(t)=\hat{U}_{\phi \bar{n}} \hat{I}_{A \bar{n}}^{\text {out }}(t) \hat{U}_{\phi \bar{n}}^{\dagger}, \\
& \hat{U}_{\phi \bar{n}}=\exp \int \frac{i d t}{e} \sum_{A} \phi_{A}(t) \hat{I}_{A \bar{n}}^{\mathrm{in}}(t), \\
& \vartheta_{n}=\int \frac{d t d t^{\prime}}{\pi} \sum_{A} \chi_{A}(t) \phi_{A}\left(t^{\prime}\right) \theta_{A n}\left(t-t^{\prime}\right) .
\end{aligned}
$$

We finally obtain

$$
\begin{aligned}
& P_{A B}(a, b)=\delta(a-b) \delta_{A B} / \tau_{A}+ \\
& \int d t \sum_{n m} \frac{\tau_{A} \delta_{A B}}{\pi^{2}} \theta_{A n}(a-t) \theta_{A m}(b-t) \\
& +\int D \phi e^{-\int \sum_{C} \frac{2 d t}{\tau_{C}} \phi_{C}^{2}(t)} \\
& \left\{\left[\frac{1}{2} \sum_{\bar{n}} \operatorname{Tr} \hat{\rho}\left(\delta \hat{I}_{A \bar{n}}^{\mathrm{in}}(a)+\delta \hat{I}_{A \bar{n}}^{\phi}(a)\right)\left(\delta \hat{I}_{B \bar{n}}^{\mathrm{in}}(b)+\delta \hat{I}_{B \bar{n}}^{\phi}(b)\right)\right.\right. \\
& \left.+\sum_{n \bar{m}} \int \frac{2 d t}{\pi} \phi_{A}(t) \theta_{A n}(a-t) \operatorname{Tr} \hat{\rho}_{B \bar{m}}^{\phi}(b)\right] \\
& +A a \leftrightarrow B b\}
\end{aligned}
$$

and

$$
\begin{aligned}
& \delta \hat{I}_{A \bar{n}}^{\mathrm{in}}(t)=-\epsilon_{A} \hat{I}_{0 \bar{n}}(t)-\hat{I}_{1 \bar{n}}(t), \\
& \delta \hat{I}_{A \bar{n}}^{\phi}(t)=\frac{e}{2 \pi}\left(T_{n} \phi_{B}^{\prime}(t)-R_{n} \phi_{A}^{\prime}(t)\right)+ \\
& \epsilon_{A} \hat{I}_{0 \bar{n}}(t)-\left(T_{n}-R_{n}\right) \hat{I}_{1 \bar{n}}(t)+2 \sqrt{R_{n} T_{n}} \hat{J}_{1 \bar{n}}^{\phi}(t), \\
& \phi_{n}(t)=\Phi(t)+\int \sqrt{2} d t^{\prime} \sum_{A} \phi_{A}\left(t+t^{\prime}\right) h_{A n}\left(\sqrt{2} t^{\prime}\right),
\end{aligned}
$$

with $B=L, R$ for $A=R, L$, respectively.

\section{Appendix D}

The third cumulant (57) has the form

$$
\langle\langle I(a) I(b) I(c)\rangle\rangle=\sum_{\bar{n}} \operatorname{Tr} \hat{\rho} \hat{F}_{\bar{n}}(a, b, c) .
$$

The operator in the last equation has the form

$$
\begin{aligned}
& \hat{F}_{\bar{n}}(a, b, c)=\sum_{\sigma(a b c)}\left[\frac{1}{6} \hat{I}_{\bar{n}}^{\Phi}\left(a_{-n}\right) \hat{I}_{\bar{n}}^{\Phi}\left(b_{-n}\right) \hat{I}_{\bar{n}}^{\Phi}\left(c_{-n}\right)\right. \\
& -\frac{1}{4}\left(\hat{I}_{1 \bar{n}}\left(a_{+n}\right) \hat{I}_{\bar{n}}^{\Phi}\left(b_{-n}\right) \hat{I}_{\bar{n}}^{\Phi}\left(c_{-n}\right)\right. \\
& \left.\left.+\hat{I}_{\bar{n}}^{\Phi}\left(a_{-n}\right) \hat{I}_{\bar{n}}^{\Phi}\left(b_{-n}\right) \hat{I}_{1 \bar{n}}\left(c_{+n}\right)\right)\right] .
\end{aligned}
$$

Here a summation over all permutations of the set $a b c$ is assumed and

$$
\hat{I}_{\bar{n}}^{\Phi}(t)=\left(R_{n}-T_{n}\right) \hat{I}_{1 \bar{n}}(t)+2 \sqrt{R_{n} T_{n}} \hat{J}_{\bar{n}}^{\Phi}(t) .
$$

In these equations $\hat{J}$ is defined by (26) and $\Phi$ by (27). Using the commutation rules (14) and (21) we get ${ }^{24.25}$

$$
\begin{aligned}
& \langle\langle I(a) I(b) I(c)\rangle\rangle=\sum_{n} R_{n} T_{n} e^{3}\left[\sum_{\sigma(a b c)} \sin \varphi\left(a_{-n}, b_{-n}\right)\right. \\
& {\left[\left(2 T_{n}-1\right) q\left(a_{-n}, c_{-n}, b_{-n}\right)+q\left(a_{-n}, c_{+n}, b_{-n}\right)\right] / 2+} \\
& \left.\left(R_{n}-T_{n}\right) \frac{e V\left(a_{-n}\right)}{\pi \hbar} \delta(a-b) \delta(a-c)\right] .
\end{aligned}
$$

The Fourier transform of $q(a, c, b)$ has the form

$$
q(\alpha, \gamma, \beta)=i \delta(\alpha+\beta+\gamma) u(\gamma)(w(\alpha)-w(\beta)),
$$

where $u$ and $w$ are defined by (23). The function $\varphi$ is defined by Eq. (43). One can also find that ${ }^{24}$

$$
\begin{aligned}
& q(a, c, b)=w(b-a) \int_{b}^{a} d s w(s-c)= \\
& \operatorname{Re} \frac{\sinh (B-A)}{\sinh ^{2}(B-A+i \epsilon)} \times \\
& \operatorname{Re} \frac{\left(k_{B} T / \hbar\right)^{3}}{2 \sinh (C-A-i \epsilon) \sinh (B-C+i \epsilon)}
\end{aligned}
$$

for $X=\pi k_{B} T x / \hbar, x=a, b, c$ and $\epsilon \rightarrow 0$. Special care must be taken at $a=b, b=c, c=a$. Note, that the function $q(a, c, b)$ is not cyclic because

$$
q(b, a, c)-q(a, c, b)=\partial_{b}[\delta(a-b) \delta(c-b)] / 2 \pi .
$$




\section{Appendix E}

In the limiting cases $\left(R_{n} \ll 1\right.$ or $\left.T_{n} \ll 1\right)$, it is possible to find expressions for the generating functional (70). Here we present some details on the derivation. The following operator expansion formula is useful,

$$
\begin{aligned}
& e^{\hat{A}+\hat{B}}=e^{\hat{A}}+\int_{0}^{1} d x e^{x \hat{A}} \hat{B} e^{(1-x) \hat{A}} \\
& +\int_{0}^{1} d x \int_{0}^{1-x} d y e^{x \hat{A}} \hat{B} e^{(1-x-y) \hat{A}} \hat{B} e^{y \hat{A}}+\ldots
\end{aligned}
$$

We use it for $\hat{A}=\left(R_{n}-T_{n}\right) \int d t i \chi\left(t_{+n}\right) \hat{I}_{1 \bar{n}}(t) / e$ and

$$
\hat{B}=2 \sqrt{R_{n} T_{n}} \int d t i \chi\left(t_{+n}\right) \hat{J}_{\bar{n}}^{\Phi}(t) / e .
$$

What we need is the term of the second power in $\hat{B}$ and an algebraic identity

$$
\begin{aligned}
& \int_{0}^{1} d x \int_{0}^{1-y} d y \xi \eta \exp [i(x-1 / 2) \xi+i(y-1 / 2) \eta]= \\
& 2 \sin \frac{\xi}{2} \sin \frac{\eta}{2}+i \sin \frac{\xi+\eta}{2}-i \frac{\xi+\eta}{\xi-\eta} \sin \frac{\xi-\eta}{2}
\end{aligned}
$$

We also need several auxiliary operator identities. Let us define

$$
\hat{E}_{\bar{n}}[\xi]=\exp \left(-\int i d t \xi(t) \hat{I}_{1 \bar{n}}(t) / e\right)
$$

and

$$
S_{0}[\xi]=\ln \operatorname{Tr} \hat{\rho} \hat{E}_{\bar{n}}^{2}[\xi]
$$

for an arbitrary function $\xi$. One can show using (14) and (21) that

$$
\int d t e^{i \omega t} \frac{\delta \operatorname{Tr} \hat{\rho} \hat{E}_{\bar{n}}^{2}[\xi]}{\delta \xi(t)}=i u(\omega) \operatorname{Tr} \hat{\rho}\left[\hat{E}_{\bar{n}}^{2}[\xi], \hat{I}_{1 \bar{n}}(\omega)\right]
$$

and

$$
\left[\hat{E}_{\bar{n}}^{2}[\xi], \hat{I}_{1 \bar{n}}(\omega)\right]=\hat{E}_{\bar{n}}^{2}[\xi] \int \frac{i \omega d t}{2 \pi} e^{i \omega t} \xi(t)
$$

So

$$
\int d t e^{i \omega t} \frac{\delta S_{0}[\xi]}{\delta \xi(t)}=-\frac{w(\omega)}{2 \pi} \int d t e^{i \omega t} \xi(t)
$$

and we finally obtain (69). Another useful property is

$$
\hat{I}_{ \pm \bar{n}}(t) \hat{E}_{\bar{n}}[\xi]=\hat{E}_{\bar{n}}[\xi] \hat{I}_{ \pm \bar{n}}(t) e^{ \pm i \xi(t)}
$$

We define the operator

$$
\hat{D}_{\bar{n}}(a, b)[\xi]=\hat{E}_{\bar{n}}[\xi] \hat{I}_{+\bar{n}}(a) \hat{I}_{-\bar{n}}(b) \hat{E}_{\bar{n}}[\xi]
$$

with $\hat{I}_{ \pm \bar{n}}(t)=\hat{I}_{2 \bar{n}}(t) \pm i \hat{I}_{3 \bar{n}}(t)$. We can show that

$$
\int d t e^{i \omega t} \frac{\delta \operatorname{Tr} \hat{\rho} \hat{D}_{\bar{n}}(a, b)[\xi]}{\delta \xi(t)}=i u(\omega) \operatorname{Tr} \hat{\rho}\left[\hat{D}_{\bar{n}}(a, b)[\xi], \hat{I}_{1 \bar{n}}(\omega)\right]
$$

and

$$
\begin{aligned}
& {\left[\hat{D}_{\bar{n}}(a, b)[\xi], \hat{I}_{1 \bar{n}}(\omega)\right]=\hat{D}_{\bar{n}}(a, b)[\xi]} \\
& \left(e^{i \omega b}-e^{i \omega a}+\int \frac{i \omega d t}{2 \pi} e^{i \omega t} \xi(t)\right) .
\end{aligned}
$$

This gives

$$
\ln \frac{\operatorname{Tr} \hat{\rho} \hat{D}_{\bar{n}}(a, b)[\xi]}{\operatorname{Tr} \hat{\rho} \hat{D}_{\bar{n}}(a, b)[0]}=S_{0}[\xi]+D(a, b)[\xi],
$$

where $D$ was defined in Eq. (71). Finally, we get

$$
S[\chi, 0]=\sum_{n} 2\left(S_{0}\left[\chi_{0 n}\right]+S_{0}\left[\chi_{1 n}\right]\right)+2 \sum_{n} S_{n}[\chi],
$$

where $2 \chi_{0 n}(t)=\chi\left(t_{-n}\right)-\chi\left(t_{+n}\right)$ and $2 \chi_{1 n}(t)=\chi\left(t_{-n}\right)+$ $\left(T_{n}-R_{n}\right) \chi\left(t_{+n}\right)$. The lowest order correction is

$$
\begin{aligned}
& S_{n}[\chi]=\int d t\left\{R_{n} T_{n} \frac{\delta S_{0}\left[\chi_{1 n}\right]}{\delta \chi_{1 n}(t)} \times\right. \\
& {\left[\frac{\chi\left(t_{+n}\right)}{T_{n}-R_{n}}-\frac{\sin \left(\left(T_{n}-R_{n}\right) \chi\left(t_{+n}\right)\right)}{\left(T_{n}-R_{n}\right)^{2}}\right]+\frac{i e V(t)}{2 \pi \hbar} \times} \\
& \left.\left[\frac{T_{n}^{2}}{T_{n}-R_{n}} \chi\left(t_{+n}\right)-R_{n} T_{n} \frac{\sin \left(\left(T_{n}-R_{n}\right) \chi\left(t_{+n}\right)\right)}{\left(T_{n}-R_{n}\right)^{2}}\right]\right\} \\
& -\frac{R_{n} T_{n}}{\left(T_{n}-R_{n}\right)^{2}} \int d t d t^{\prime} \frac{w\left(t-t^{\prime}\right)}{\pi} e^{i \varphi\left(t^{\prime}, t\right)+D\left(t^{\prime}, t\right)\left[\chi_{1 n}\right]} \times \\
& \sin \frac{\left(T_{n}-R_{n}\right) \chi\left(t_{+n}\right)}{2} \sin \frac{\left(T_{n}-R_{n}\right) \chi\left(t_{+n}^{\prime}\right)}{2} .
\end{aligned}
$$

We stress that the above formula are exact up to first order in $T_{n} \ll 1$ or $R_{n} \ll 1$. After taking the respective limit higher order contributions should be disregarded as we would need also $\hat{B}^{4}$-terms.

Note also that $\hat{I}_{\bar{o} \bar{n}}=\left(R_{n}-T_{n}\right) \hat{I}_{1 \bar{n}}+2 \sqrt{R_{n} T_{n}} \hat{I}_{2 \bar{n}}$ satisfies the same commutation rules with itself and the Hamiltonian as $\hat{I}_{1 \bar{n}}$. This leads to the identity

$$
\begin{aligned}
& 0=\int d t d t^{\prime} w\left(t-t^{\prime}\right) \times \\
& \left\{\chi\left(t^{\prime}\right) \sin \chi(t)-4 \sin \frac{\chi(t)}{2} \sin \frac{\chi\left(t^{\prime}\right)}{2} e^{D\left(t, t^{\prime}\right)[\chi]}\right\}
\end{aligned}
$$

for a sufficiently regular function $\chi$.

\section{Appendix F}

In the bosonized version (appendix A), we can write in (76)

$$
\begin{aligned}
& \hat{H}_{d}=\int d s i \hbar\left[\hat{\psi}_{l}^{\dagger}(s) \partial_{s} \hat{\psi}_{l}(s)-\hat{\psi}_{r}^{\dagger}(s) \partial_{s} \hat{\psi}_{r}(s)\right] \\
& \hat{I}_{d}(s)=e\left(\hat{\psi}_{r}^{\dagger}(s) \hat{\psi}_{r}(s)-\hat{\psi}_{l}^{\dagger}(s) \hat{\psi}_{l}(s)\right) \\
& \hat{Q}_{d}=e\left(\hat{\psi}_{r}^{\dagger}(s) \hat{\psi}_{r}(s)+\hat{\psi}_{l}^{\dagger}(s) \hat{\psi}_{l}(s)\right)
\end{aligned}
$$


with $\left\{\hat{\psi}_{a}(s), \hat{\psi}_{b}^{\dagger}\left(s^{\prime}\right)\right\}=\delta_{a b} \delta\left(s-s^{\prime}\right)$ for $a, b=l, r$. To derive (81), we first introduce the auxiliary decomposition

$$
\hat{I}_{d}(s)=\hat{I}_{r}(s)-\hat{I}_{l}(s), \hat{Q}_{d}(s)=\hat{I}_{r}(s)+\hat{I}_{l}(s)
$$

with the commutation rules

$$
\begin{aligned}
& {\left[\hat{I}_{l}(s), \hat{I}_{r}\left(s^{\prime}\right)\right]=0,} \\
& {\left[\hat{I}_{l}(s), \hat{I}_{l}\left(s^{\prime}\right)\right]=\left[\hat{I}_{r}\left(s^{\prime}\right), \hat{I}_{r}(s)\right]=i e^{2} \partial_{s}\left(s-s^{\prime}\right) / 2 \pi}
\end{aligned}
$$

The last useful set of commutators is

$$
\begin{aligned}
& {\left[\hat{H}_{d}, \hat{Q}_{d}(s)\right]=i \hbar \partial_{s} \hat{I}_{d}(s),} \\
& {\left[\hat{H}_{d}, \hat{I}_{d}(s)\right]=-i \hbar \partial_{s} \hat{Q}_{d}(s),} \\
& {\left[\hat{H}_{d}, \hat{I}_{r}(s)\right]=i \hbar \partial_{s} \hat{I}_{r}(s),} \\
& {\left[\hat{H}_{d}, \hat{I}_{l}(s)\right]=-i \hbar \partial_{s} \hat{I}_{l}(s) .}
\end{aligned}
$$

Similarly to (22), equilibrium averages for the decoupled detector $(\lambda=0)$ are

$$
\begin{aligned}
& \operatorname{Tr} \hat{\rho}_{d} \hat{I}_{d}(\omega)=0, \\
& \operatorname{Tr} \hat{\rho}_{d} \hat{Q}_{d}(\omega)=2 \pi n \delta(\omega), \\
& \operatorname{Tr} \hat{\rho}_{d} \hat{I}_{l}(\alpha) \hat{I}_{r}(\beta)=0, \\
& \operatorname{Tr} \hat{\rho}_{d} \hat{I}_{l}(\alpha) \hat{I}_{l}(\beta)=\operatorname{Tr} \hat{\rho}_{d} \hat{I}_{r}(\beta) \hat{I}_{r}(\alpha)= \\
& =\frac{e^{2}}{2} \delta(\alpha+\beta)\left(w_{d}(\alpha)+\alpha\right),
\end{aligned}
$$

where $n$ is average charge density and $w_{d}(\omega)=$ $\omega \operatorname{coth}\left(\hbar \omega / k_{B} T_{d}\right)$. We shall put $n=0$ assuming that the average charge is screened out. In the Heisenberg picture, we have

$$
\begin{aligned}
& \left(\partial_{t}-\partial_{s}\right) \hat{I}_{l}(s, t)=-\partial_{s} \lambda(s) \hat{Q}(t), \\
& \left(\partial_{t}+\partial_{s}\right) \hat{I}_{r}(s, t)=\partial_{s} \lambda(s) \hat{Q}(t) .
\end{aligned}
$$

The general solution is

$$
\begin{aligned}
& \hat{I}_{l}(s, t)=\hat{I}_{l}(t+s)-\partial_{s} \int_{s}^{\infty} d s^{\prime} \lambda\left(s^{\prime}\right) \hat{Q}\left(t+s-s^{\prime}\right), \\
& \hat{I}_{r}(s, t)=\hat{I}_{r}(s-t)+\partial_{s} \int_{-\infty}^{s} d s^{\prime} \lambda\left(s^{\prime}\right) \hat{Q}\left(t-s+s^{\prime}\right) .
\end{aligned}
$$

Hence, we have in the Heisenberg picture

$$
\begin{aligned}
& 2 \hat{\tilde{I}}(s, t)=2 \partial_{s} \int d s^{\prime} \lambda\left(s^{\prime}\right) \hat{Q}\left(t-\left|s-s^{\prime}\right|\right)+ \\
& \hat{I}_{r}(s-t)-\hat{I}_{r}(-s-t)-\hat{I}_{l}(t+s)+\hat{I}_{l}(t-s)
\end{aligned}
$$

1 A.D. Stone, P.A. Mello, K.A. Muttalib, J.-L. Pichard, in: B.L. Altshuler, P.A. Lee, R.A. Webb (Eds.), Mesoscopic Phenomena in Solids (North-Holland, Amsterdam, 1991).

2 Semiconductor Spintronics and Quantum Computation, edited by D. D. Awschalom, D. Loss, and N. Samarth and

$$
\begin{aligned}
& \int d s \lambda(s) \hat{Q}_{d}(s, t)=\int d s \lambda(s)\left(\hat{I}_{r}(s-t)+\hat{I}_{l}(s+t)\right) \\
& -2 \int_{0}^{\infty} d s \int d s^{\prime} \lambda\left(s^{\prime}\right) \lambda^{\prime}\left(s-s^{\prime}\right) \hat{Q}(t-s) .
\end{aligned}
$$

To find $\mathcal{S}$, we need the Keldysh generating functional, $\stackrel{40}{=}$

$$
\begin{aligned}
& e^{S[\chi, \phi]}=\operatorname{Tr} \hat{\rho} \times \\
& \tilde{\mathcal{T}} e^{\int i d t\left[\chi_{Q}^{-}(t) \hat{Q}(t)+\int d s\left(\chi_{r}^{-}(s, t) \hat{I}_{r}(s, t)+\chi_{l}^{-}(s, t) \hat{I}_{l}(s, t)\right)\right]} \times \\
& \mathcal{T} e^{\int i d t\left[\chi_{Q}^{+}(t) \hat{Q}(t)+\int d s\left(\chi_{r}^{+}(s, t) \hat{I}_{r}(s, t)+\chi_{l}^{+}(s, t) \hat{I}_{l}(s, t)\right)\right]}
\end{aligned}
$$

where $\chi^{ \pm}=\chi / 2 \pm \phi$ and $S[0, \phi]=0$. The functional is related to a quasiprobability in presence of external fields

$$
\begin{aligned}
& \varrho\left[I_{r}, I_{l}, Q ; \phi\right]=\int D \chi e^{S[\chi, \phi]} \times \\
& e^{-\int i d t\left[\chi_{Q}(t) Q(t)+\int d s\left(\chi_{r}(s, t) I_{r}(s, t)+\chi_{l}(s, t) I_{l}(s, t)\right)\right]}
\end{aligned}
$$

One can show that Heisenberg equations (F6) and (F7) are satisfied for the quasiprobability and $I_{r}, I_{l}$ and $Q$ instead of the corresponding operators. It can be also shown that the backaction of the detector on the system can be simplified by the classical mapping $\hat{Q} \rightarrow Q, \hat{Q}_{d} \rightarrow$ $Q_{d}$ in Hamiltonian (76) used in (

$$
\hat{H}_{I}(t)=-2 \pi \hbar \int d s \lambda(s)\left(Q_{d}(s, t) \hat{Q}+\hat{Q}_{d}(s, t) Q(t)\right) / e^{2} .
$$

The final generating functional reads

$$
\begin{aligned}
& e^{\mathcal{S}[\chi]}=\int D \phi D \xi D \eta e^{\int i d t(\xi(t) \eta(t)-\chi(t) \phi(t) / 2)} \times \\
& e^{-\int d \omega\left[|\chi(\omega)|^{2} w_{d}(\omega) / 32 \pi^{2}+|\phi(\omega)|^{2} / 2 w_{d}(\omega)\right]} \times \\
& e^{\tilde{S}[-\dot{\chi} * \lambda-\xi,-2 \pi \phi * \lambda+4 \pi \tilde{\eta}]}
\end{aligned}
$$

with $\tilde{S}$ defined by (82) and $\tilde{\eta}(t)=\int_{0}^{\infty} d s \dot{\lambda} * \lambda(s) \eta(t-s)$, $w_{d}(\omega)=\omega \operatorname{coth}\left(\hbar \omega / k_{B} T_{d}\right)$. In the case of many tapes, (93) follows from

$$
\begin{aligned}
& e^{\mathcal{S}[\chi]}=\int D \phi D \xi D \eta e^{\int i d t\left(\xi(t) \eta(t)-\chi(t) \sum_{c} \phi_{c}(t) / 2\right)} \times \\
& e^{-\int d \omega\left[|\chi(\omega)|^{2} \sum_{c} w_{c}(\omega) / 32 \pi^{2}+\sum_{c}\left|\phi_{c}(\omega)\right|^{2} / 2 w_{c}(\omega)\right]} \times \\
& e^{\tilde{S}\left[-\xi-\sum_{c} \dot{\chi}^{*} * \lambda_{c},-2 \pi \sum_{c} \phi_{c} * \lambda_{c}+4 \pi \tilde{\eta}\right]}
\end{aligned}
$$

with $\tilde{\eta}(t)=\sum_{c} \int_{0}^{\infty} d s \dot{\lambda}_{c} * \lambda_{c}(s) \eta(t-s)$.

(Springer, Berlin, 2002).

3 J. von Neumann, Mathematical Foundations of Quantum Mechanics (Princeton U.P., Princeton, 1932).

4 for a review, see Y.M. Blanter and M. Büttiker, Phys. Rep. 336, 1 (2000); Y. V. Nazarov and Y. M. Blanter Quantum 
Transport (Cambridge University Press, 2009).

5 M. I. Reznikov et al., Phys. Rev. Lett. 753340 (1995); A. Kumar et al., Phys. Rev. Lett. 76, 2778 (1996).

${ }^{6}$ L.S. Levitov and G.B. Lesovik, JETP Lett. 58, 230 (1993); L.S. Levitov, H.W. Lee, G.B. Lesovik, J. Math. Phys. 37, 4345 (1996); W. Belzig and Y.V. Nazarov, Phys. Rev. Lett. 87, 197006 (2001); W. Belzig and Yu. V. Nazarov, Phys. Rev. Lett. 87, 067006 (2001); Y.V. Nazarov and M. Kindermann, Eur. J. Phys. B 35, 413 (2003); M. Kindermann and Y.V. Nazarov in Ref. 7; I. Snyman and Y. V. Nazarov, Phys. Rev. B 77, 165118 (2008).

7 Quantum Noise in Mesoscopic Physics, Y.V. Nazarov (Ed.), (Kluwer, Dordrecht, 2003).

8 B. Reulet et al., Phys. Rev. Lett. 91, 196601 (2003).

9 Y. Bomze et al., Phys. Rev. Lett. 95, 176601 (2005); 101, 016803 (2008).

10 C.W.J. Beenakker, M. Kindermann, and Yu.V. Nazarov, Phys. Rev. Lett. 90176802 (2003); Phys. Rev. B 69, 035336 (2004).

11 R. J. Schoelkopf et al. in Ref. 7.

12 R.J. Schoelkopf et al., Phys. Rev. Lett. 78, 3370 (1997).

13 E. Zakka-Bajjani et al., Phys. Rev. Lett. 99, 236803 (2007).

14 J. Gabelli and B. Reulet, Phys. Rev. Lett. 100, 026601 (2008).

15 B. Misra and E. C. G. Sudarshan, J. Math. Phys. (N.Y.) 18, 756 (1977).

16 V. B. Braginsky and F. Ya. Khalili, Quantum Measurement (Cambridge University Press, Cambridge, 1992).

17 A. N. Jordan and M. Büttiker, Phys. Rev. B 71, 125333 (2005); A. N. Jordan, A. N. Korotkov, and M. Büttiker, Phys. Rev. Lett. 97, 026805 (2006); A.N. Jordan and A.N. Korotkov, Phys. Rev. B 74, 085307 (2006); A. N. Korotkov in Ref. 7 .

18 D.V. Averin, K. Rabenstein and V.K. Semenov, Phys. Rev. B 73, 094504 (2006).

19 K. V. Bayandin, A.V. Lebedev, G. B. Lesovik, JETP 106, 117 (2008).

${ }^{20}$ K. Kraus, States, Effects and Operations (Springer, Berlin, 1983).
21 See e. g. J. Preskill, Quantum Information and Computation (www.theory.caltech.edu/people/preskill/ph229)

22 M. A. Naimark, Izv. Akad. Nauk. SSSR, Ser. Mat. 4, 277 (1940).

23 We thank Peter Hänggi for reminding us on this issue.

24 A.V. Galaktionov, D.S. Golubev and A.D. Zaikin, Phys. Rev. B. 68, 235333 (2003); 72, 205417 (2005).

25 J. Salo, F.W.J. Hekking, and J.P. Pekola, Phys. Rev. B 74, 125427 (2006).

26 T. T. Heikkilä and T. Ojanen, Phys. Rev. B 75, 035335 (2007).

27 J. Gabelli and B. Reulet, J. Stat. Mech. P01049 (2009).

28 C.W.J. Beenakker, H. Schomerus, Phys.Rev.Lett. 86, 700 (2001); 93, 096801 (2004).

29 J. Gabelli, L.-H. Reydellet, G. Fe've, J.-M. Berroir, B. Placais, P. Roche, and D. C. Glattli, Phys. Rev. Lett. 93, 056801 (2004).

30 See e.g. T. Giamarchi, Quantum Physics in One Dimension (Claverdon Press, Oxford, 2004).

31 A. Bednorz and W. Belzig, Phys. Rev. Lett. 101, 206803 (2008).

32 J. Tobiska and Y.V. Nazarov, Phys. Rev. B 72, 235328 (2005).

33 U. Gavish, Y. Imry, and B. Yurke, SPIE Proceedings Vol. 5469 (SPIE-International Society for Optical Engineering, Bellingham, WA, 2004), p. 257; Phys. Rev. Lett. 93, 250601 (2004).

34 G.B. Lesovik and L.S. Levitov, Phys. Rev. Lett. 72, 538 (1994)

35 L. S. Levitov and M. Reznikov, Phys. Rev. B 70, 115305 (2004).

36 M. J. M. de Jong, Phys. Rev. B 54, 8144 (1996).

37 W. Belzig, in Ref. 7

38 D. A. Bagrets and Yu. V. Nazarov, Phys. Rev. B 67, 085316 (2003).

39 F. Pistolesi, Phys. Rev. B 69, 245409 (2004).

40 A. Kamenev, A. Levchenko, Advances in Physics 58, 197 (2009). 